\title{
General Decay and Blow-Up of Solutions for a System of Viscoelastic Equations of Kirchhoff Type with Strong Damping
}

\author{
Wenjun Liu, Gang Li, and Linghui Hong \\ College of Mathematics and Statistics, Nanjing University of Information Science and Technology, Nanjing 210044, China \\ Correspondence should be addressed to Wenjun Liu; wjliu@nuist.edu.cn
}

Received 8 May 2013; Revised 28 September 2013; Accepted 16 November 2013; Published 4 February 2014

Academic Editor: William Ziemer

Copyright (C) 2014 Wenjun Liu et al. This is an open access article distributed under the Creative Commons Attribution License, which permits unrestricted use, distribution, and reproduction in any medium, provided the original work is properly cited.

\begin{abstract}
The general decay and blow-up of solutions for a system of viscoelastic equations of Kirchhoff type with strong damping is considered. We first establish two blow-up results: one is for certain solutions with nonpositive initial energy as well as positive initial energy by exploiting the convexity technique, the other is for certain solutions with arbitrarily positive initial energy based on the method of Li and Tsai. Then, we give a decay result of global solutions by the perturbed energy method under a weaker assumption on the relaxation functions.
\end{abstract}

\section{Introduction}

In this work, we investigate the following system of viscoelastic equations of Kirchhoff type:

$$
\begin{gathered}
u_{t t}-M\left(\|\nabla u\|_{2}^{2}\right) \Delta u+\int_{0}^{t} g_{1}(t-s) \Delta u(s) \mathrm{d} s-\Delta u_{t} \\
=f_{1}(u, v), \quad(x, t) \in \Omega \times(0, \infty), \\
v_{t t}-M\left(\|\nabla v\|_{2}^{2}\right) \Delta v+\int_{0}^{t} g_{2}(t-s) \Delta v(s) \mathrm{d} s-\Delta v_{t} \\
=f_{2}(u, v), \quad(x, t) \in \Omega \times(0, \infty), \\
u(x, t)=0, \quad v(x, t)=0, \\
\quad(x, t) \in \partial \Omega \times[0, \infty), \\
\begin{aligned}
u(x, 0)=u_{0}(x), \quad u_{t}(x, 0)=u_{1}(x), \quad x \in \Omega, \\
v(x, 0)=v_{0}(x), \quad v_{t}(x, 0)=v_{1}(x), \quad x \in \Omega,
\end{aligned}
\end{gathered}
$$

where $\Omega \subset \mathbb{R}^{n}, n \geq 1$, is a bounded domain with smooth boundary $\partial \Omega, M$ is a positive locally Lipschitz function, and $g_{i}(\cdot): \mathbb{R}^{+} \rightarrow \mathbb{R}^{+}(i=1,2),\left(f_{1}, f_{2}\right): \mathbb{R}^{2} \rightarrow \mathbb{R}^{2}$ are given functions to be specified later.
To motivate our work, let us recall some previous results regarding viscoelastic equations of Kirchhoff type. The following problem:

$$
\begin{array}{r}
u_{t t}-M\left(\|\nabla u\|_{2}^{2}\right) \Delta u+\int_{0}^{t} g(t-s) \Delta u(s) \mathrm{d} s+h\left(u_{t}\right)=f(u), \\
(x, t) \in \Omega \times(0, \infty), \\
u=0, \quad(x, t) \in \partial \Omega \times[0, \infty), \\
u(x, 0)=u_{0}(x), \quad u_{t}(x, 0)=u_{1}(x), \quad x \in \Omega
\end{array}
$$

is a model to describe the motion of deformable solids as hereditary effect is incorporated. It was first studied by Torrejón and Yong [1] who proved the existence of a weakly asymptotic stable solution for large analytical datum. Later, Muñoz Rivera [2] showed the existence of global solutions for small datum and the total energy decays to zero exponentially under some restrictions. Then, $\mathrm{Wu}$ and Tsai [3] treated problem (2) for $h\left(u_{t}\right)=-\Delta u_{t}$ and proved the global existence, decay, and blow-up with suitable conditions on initial data. They obtained the blow-up properties of local solution with small positive initial energy by the direct method of [4]. To obtain the decay result, they assumed that the nonnegative kernel $g^{\prime}(t) \leq-r g(t), \forall t \geq 0$ for some $r>$ 0 . This energy decay result was recently improved by $\mathrm{Wu}$ 
in [5] under a weaker condition on $g$ (i.e., $g^{\prime}(t) \leq 0$ for $t \geq 0$ ). For a single wave equation of Kirchhoff type without the viscoelastic term, we refer the reader to Matsuyama and Ikehata [6] and Ono [7-10].

Many results concerning local existence, global existence, decay, and blow-up of solutions for a system of wave equations of Kirchhoff type without viscoelastic terms (i.e., $\left.g_{i}=0, i=1,2\right)$ have also been extensively studied. For example, Park and Bae [11] considered the system of wave equations with nonlinear dampings for $f_{1}(u, v)=$ $\mu|u|^{q-1} u$ and $f_{2}(u, v)=\mu|v|^{q-1} v, q \geq 1$, and showed the global existence and asymptotic behavior of solutions under some restrictions on the initial energy. Later, Benaissa and Messaoudi [12] discussed blow-up properties for negative initial energy. Recently, Wu and Tsai [13] studied the system (1) for $g_{i}=0(i=1,2)$. Under some suitable assumptions on $f_{i}(i=1,2)$, they proved local existence of solutions by applying the Banach fixed point theorem and the blow-up of solutions by using the method of $\mathrm{Li}$ and Tsai in [4], where three different cases on the sign of the initial energy $E(0)$ are considered.

In the case of $M \equiv 1$ and in the presence of viscoelastic term (i.e., $g \neq 0$ ), Cavalcanti et al. [14] studied the equation that was subject to a locally distributed dissipation

$$
\begin{array}{r}
u_{t t}-\Delta u+\int_{0}^{t} g(t-s) \Delta u(s) \mathrm{d} s+a(x) u_{t}+|u|^{\gamma} u=0, \\
(x, t) \in \Omega \times(0, \infty),
\end{array}
$$

with the same initial and boundary conditions as that of (2), and proved an exponential decay rate. This work extended the result of Zuazua [15], in which he considered (3) with $g=0$ and the localized linear damping. By using the piecewise multipliers method, Cavalcanti and Oquendo [16] investigated the equation

$$
\begin{aligned}
u_{t t} & -k_{0} \Delta u+\int_{0}^{t} \operatorname{div}[a(x) g(t-s) \nabla u(s)] \mathrm{d} s \\
& +b(x) h\left(u_{t}\right)+f(u)=0, \quad(x, t) \in \Omega \times(0, \infty),
\end{aligned}
$$

with the same initial and boundary conditions as that of (2). Under the similar conditions on the relaxation function $g$ as above, and $a(x)+b(x) \geq \delta>0$ for all $x \in \Omega$, they improved the results of [14] by establishing exponential stability for exponential decay function $g$ and linear function $h$, and polynomial stability for polynomial decay function $g$ and nonlinear function $h$, respectively.

Concerning blow-up results, Messaoudi [17] considered the equation

$$
\begin{array}{r}
u_{t t}-\Delta u+\int_{0}^{t} g(t-s) \Delta u(s) \mathrm{d} s+a u_{t}\left|u_{t}\right|^{m-2}=b|u|^{r-2} u, \\
(x, t) \in \Omega \times(0, \infty) .
\end{array}
$$

He proved that any weak solution with negative initial energy blows up in finite time if $r>m$ and

$$
\int_{0}^{\infty} g(s) \mathrm{d} s \leq \frac{r-2}{r-2+1 / r}
$$

while exists globally for any initial data in the appropriate space if $m \geq r$. This result was improved by the same author in [18] for positive initial energy under suitable conditions on $g$, $m$, and $r$. Recently, Liu [19] studied the equation

$$
\begin{array}{r}
u_{t t}-\Delta u+\int_{0}^{t} g(t-s) \Delta u(s) \mathrm{d} s-\omega \Delta u_{t}+\mu u_{t}=|u|^{r-2} u, \\
(x, t) \in \Omega \times(0, \infty),
\end{array}
$$

with the same initial and boundary condition as that of (2). By virtue of convexity technique and supposing that

$$
\int_{0}^{\infty} g(s) \mathrm{d} s \leq \frac{r-2}{r-2+1 /\left[(1-\hat{\delta})^{2} r+2 \delta(1-\hat{\delta})\right]},
$$

where $\widehat{\delta}=\max \{0, \delta\}$, he proved that the solution with nonpositive initial energy as well as positive initial energy blows up in finite time.

We should mention that the following system:

$$
\begin{gathered}
u_{t t}-\Delta u+\int_{0}^{t} g_{1}(t-\tau) \Delta u(\tau) \mathrm{d} \tau+\left|u_{t}\right|^{m-1} u_{t}=f_{1}(u, v), \\
(x, t) \in \Omega \times(0, T), \\
v_{t t}-\Delta v+\int_{0}^{t} g_{2}(t-\tau) \Delta v(\tau) \mathrm{d} \tau+\left|v_{t}\right|^{\gamma-1} v_{t}=f_{2}(u, v), \\
(x, t) \in \Omega \times(0, T), \\
u(x, t)=0, \quad v(x, t)=0, \\
\begin{array}{c}
u(x, 0)=u_{0}(x), \quad u_{t}(x, 0)=u_{1}(x), \quad x \in \Omega, \\
v(x, 0)=v_{0}(x), \quad v_{t}(x, 0)=v_{1}(x), \quad x \in \Omega
\end{array}
\end{gathered}
$$

was considered by Han and Wang in [20], where $\Omega$ is a bounded domain with smooth boundary $\partial \Omega$ in $\mathbb{R}^{n}, n=$ $1,2,3$. Under suitable assumptions on the functions $g_{i}, f_{i}(i=$ $1,2)$, the initial data and the parameters in the above problem established local existence, global existence, and blow-up property (the initial energy $E(0)<0$ ). This latter blow-up result has been improved by Messaoudi and Said-Houari [21] into certain solutions with positive initial energy. Recently, Liang and Gao in [22] investigated the following problem:

$$
\begin{array}{r}
u_{t t}-\Delta u+\int_{0}^{t} g_{1}(t-\tau) \Delta u(\tau) \mathrm{d} \tau-\Delta u_{t}=f_{1}(u, v), \\
(x, t) \in \Omega \times(0, T), \\
v_{t t}-\Delta v+\int_{0}^{t} g_{2}(t-\tau) \Delta v(\tau) \mathrm{d} \tau-\Delta v_{t}=f_{2}(u, v), \\
(x, t) \in \Omega \times(0, T),
\end{array}
$$

with the same initial and boundary conditions as that of (9). Under suitable assumptions on the functions $g_{i}, f_{i}(i=1,2)$ 
and certain initial data in the stable set, they proved that the decay rate of the solution energy is exponential. Conversely, for certain initial data in the unstable set, they proved that there are solutions with positive initial energy that blow up in finite time. It is also worth mentioning the work [23] in which we studied system (1). Under suitable assumptions on the functions $g_{i}, f_{i}(i=1,2)$ and certain initial conditions, we showed that the solutions are global in time and the energy decays exponentially. For other papers related to existence, uniform decay, and blow-up of solutions of nonlinear wave equations, we refer the reader to [14, 24-29] for existence and uniform decay, to [17, 30-34] for blow-up, and to [35-40] for the coupled system. To the best of our knowledge, the general decay and blow-up of solutions for systems of viscoelastic equations of Kirchhoff type with strong damping have not been well studied.

Motivated by the above mentioned research, we consider in the present work the coupled system (1) with nonzero $g_{i}(i=1,2)$ and nonconstant $M(s)$. We note that in such a coupled system case we should overcome the additional difficulties brought by the treatment of the nonlinear coupled terms. We first establish two blow-up results: one is for certain solutions with nonpositive initial energy as well as positive initial energy, the other is for certain solutions with arbitrarily positive initial energy. Then, we give a decay result of global solutions under a weaker assumption on the relaxation functions $g_{i}(t)(i=1,2)$.

This paper is organized as follows. In the next section we present some assumptions, notations and known results and state the main results: Theorems 4, 5, 6, and 7. The two blowup results, Theorems 5 and 6, are proved in Sections 3 and 4 , respectively. Section 5 is devoted to the proof of the decay result-Theorem 7 .

\section{Preliminaries and Main Result}

In this section we present some assumptions, notations, and known results and state the main results. First, we make the following assumptions.

(A1) $M(s)$ is a positive locally Lipschitz function for $s \geq$ 0 with the Lipschitz constant $L$ satisfying

$$
M(s) \geq m_{0}>0 .
$$

$(\mathrm{A} 2) g_{i}(t):[0, \infty) \rightarrow(0, \infty),(i=1,2)$ are strictly decreasing $C^{1}$ functions such that

$$
m_{0}-\int_{0}^{\infty} g_{i}(s) \mathrm{d} s=l_{i}>0 .
$$

(A3) There exist two positive differentiable functions $\xi_{1}(t)$ and $\xi_{2}(t)$ such that

$$
g_{i}^{\prime}(t) \leq-\xi_{i}(t) g_{i}(t), \quad(i=1,2), \text { for } t \geq 0,
$$

and $\xi_{i}(t)$ satisfies

$$
\xi_{i}^{\prime}(t) \leq 0, \quad \forall t>0, \quad \int_{0}^{+\infty} \xi_{i}(t) \mathrm{d} t=\infty, \quad(i=1,2) .
$$

(A4) We make the following extra assumption on $M$ :

$$
\begin{gathered}
2(p+2) \bar{M}(s)-2 M(s) s \geq 2(p+1) m_{0} s, \quad \forall s \geq 0, \\
\text { where } \bar{M}(s)=\int_{0}^{s} M(\tau) \mathrm{d} \tau .
\end{gathered}
$$

Remark 1. It is clear that $M(s)=m_{0}+m_{1} s^{\gamma}$ (which occurs physically in the study of vibrations of damped flexible space structures in a bounded domain in $\mathbb{R}^{n}$ ) satisfies (A4) for $s \geq$ $0, m_{0}>0, m_{1} \geq 0, \gamma \geq 0$ as long as $p+1>\gamma$. Indeed, by straightforward calculations, we obtain

$$
\begin{aligned}
2(p+ & 2) \bar{M}(s)-2 M(s) s \\
& =2(p+2)\left(m_{0} s+\frac{m_{1}}{\gamma+1} s^{\gamma+1}\right)-2 m_{0} s-2 m_{1} s^{\gamma+1} \\
& =2(p+1) m_{0} s+\frac{2(p+1-\gamma)}{\gamma+1} m_{1} s^{\gamma+1} \geq 2(p+1) m_{0} s .
\end{aligned}
$$

Next, we introduce some notations. Consider

$$
\begin{aligned}
\left(g_{i} \diamond \nabla w\right)(t) & =\int_{0}^{t} g_{i}(t-s)\|\nabla w(t)-\nabla w(s)\|_{2}^{2} \mathrm{~d} s, \\
l & =\min \left\{l_{1}, l_{2}\right\}, \\
g_{0}(t) & =\max \left\{g_{1}(t), g_{2}(t)\right\}, \quad \forall t \geq 0, \\
\|\cdot\|_{q} & =\|\cdot\|_{L^{q}(\Omega)}, \quad 1 \leq q \leq \infty,
\end{aligned}
$$

the Hilbert space $L^{2}(\Omega)$ endowed with the inner product

$$
(u, v)=\int_{\Omega} u(x) v(x) \mathrm{d} x
$$

and the functions $f_{1}(u, v)$ and $f_{2}(u, v)$ (see also [21]):

$$
\begin{aligned}
& f_{1}(u, v)=\left[a|u+v|^{2(p+1)}(u+v)+b|u|^{p} u|v|^{p+2}\right], \\
& f_{2}(u, v)=\left[a|u+v|^{2(p+1)}(u+v)+b|u|^{p+2}|v|^{p} v\right],
\end{aligned}
$$

where $a, b>0$ are constants and $p$ satisfies

$$
\begin{aligned}
& -1<p, \quad \text { if } n=1,2, \\
& -1<p \leq \frac{(3-n)}{(n-2)}, \quad \text { if } n \geq 3 .
\end{aligned}
$$

One can easily verify that

$$
u f_{1}(u, v)+v f_{2}(u, v)=2(p+2) F(u, v), \quad \forall(u, v) \in \mathbb{R}^{2},
$$

where

$$
F(u, v)=\frac{1}{2(p+2)}\left[a|u+v|^{2(p+2)}+2 b|u v|^{p+2}\right] .
$$

Then, we give two lemmas which will be used throughout this work. 
Lemma 2 (Sobolev-Poincaré inequality [41]). If $2 \leq \rho \leq$ $2 n /(n-2)$, then

$$
\|u\|_{\rho} \leq C\|\nabla u\|_{2} \quad \text { for } u \in H_{0}^{1}(\Omega)
$$

holds with some constant $C$.

Lemma 3 ([21, Lemma 3.2]). Assume that (20) holds. Then there exists $\eta_{1}>0$ such that for any $(u, v) \in\left(H_{0}^{1}(\Omega) \cap\right.$ $\left.H^{2}(\Omega)\right) \times\left(H_{0}^{1}(\Omega) \cap H^{2}(\Omega)\right)$, one has

$$
\begin{aligned}
a \| u+ & v\left\|_{2(p+2)}^{2(p+2)}+2 b\right\| u v \|_{p+2}^{p+2} \\
& \leq \eta_{1}\left(l_{1}\|\nabla u\|_{2}^{2}+l_{2}\|\nabla v\|_{2}^{2}\right)^{p+2} .
\end{aligned}
$$

We now state a local existence theorem for system (1), whose proof follows the arguments in $[3,13]$.

Theorem 4. Suppose that (20), (A1), and (A2) hold, and that $u_{0}, v_{0} \in H_{0}^{1}(\Omega) \cap H^{2}(\Omega)$ and $u_{1}, v_{1} \in L^{2}(\Omega)$. Then problem (1) has a unique local solution

$$
\begin{aligned}
& u, v \in C\left([0, T) ; H_{0}^{1}(\Omega) \cap H^{2}(\Omega)\right), \\
& u_{t}, v_{t} \in C\left([0, T) ; L^{2}(\Omega)\right) \cap L^{2}\left([0, T) ; H_{0}^{1}(\Omega)\right),
\end{aligned}
$$

for some $T>0$. Moreover, at least one of the following statements is valid:

$$
\begin{aligned}
& \text { (1) } T=\infty \\
& \text { (2) } \lim _{t \rightarrow T^{-}}\left(\left\|u_{t}\right\|_{2}^{2}+\left\|v_{t}\right\|_{2}^{2}+\|\Delta u\|_{2}^{2}+\|\Delta v\|_{2}^{2}\right)=\infty .
\end{aligned}
$$

The energy associated with system (1) is given by

$$
\begin{aligned}
E(t)= & \frac{1}{2}\left(\left\|u_{t}\right\|_{2}^{2}+\left\|v_{t}\right\|_{2}^{2}\right)-\frac{1}{2}\left(\int_{0}^{t} g_{1}(s) \mathrm{d} s\right)\|\nabla u\|_{2}^{2} \\
& -\frac{1}{2}\left(\int_{0}^{t} g_{2}(s) \mathrm{d} s\right)\|\nabla v\|_{2}^{2}-\int_{\Omega} F(u, v) \mathrm{d} x \\
& +\frac{1}{2}\left[\left(g_{1} \diamond \nabla u\right)(t)+\left(g_{2} \diamond \nabla v\right)(t)\right] \\
& +\frac{1}{2} \bar{M}\left(\|\nabla u\|_{2}^{2}\right)+\frac{1}{2} \bar{M}\left(\|\nabla v\|_{2}^{2}\right), \quad \text { for } t \geq 0 .
\end{aligned}
$$

As in [5], we can get

$$
\begin{aligned}
& E^{\prime}(t) \\
& =-\left(\left\|\nabla u_{t}\right\|_{2}^{2}+\left\|\nabla v_{t}\right\|_{2}^{2}\right)-\frac{1}{2}\left[g_{1}(t)\|\nabla u\|_{2}^{2}+g_{2}(t)\|\nabla v\|_{2}^{2}\right] \\
& \quad+\frac{1}{2}\left[\left(g_{1}^{\prime} \diamond \nabla u\right)(t)+\left(g_{2}^{\prime} \diamond \nabla v\right)(t)\right] \leq 0, \quad \forall t \geq 0 .
\end{aligned}
$$

Then we have

$$
\begin{aligned}
E(t)= & E(0)-\int_{0}^{t}\left(\left\|\nabla u_{t}(\tau)\right\|_{2}^{2}+\left\|\nabla v_{t}(\tau)\right\|_{2}^{2}\right) \mathrm{d} \tau \\
& -\frac{1}{2} \int_{0}^{t}\left[g_{1}(\tau)\|\nabla u(\tau)\|_{2}^{2}+g_{2}(\tau)\|\nabla v(\tau)\|_{2}^{2}\right] \mathrm{d} \tau \\
& +\frac{1}{2} \int_{0}^{t}\left[\left(g_{1}^{\prime} \diamond \nabla u\right)(\tau)+\left(g_{2}^{\prime} \diamond \nabla v\right)(\tau)\right] \mathrm{d} \tau .
\end{aligned}
$$

We introduce

$$
\begin{aligned}
& B_{1}=\eta_{1}^{1 / 2(p+2)}, \quad \alpha_{*}=B_{1}^{-(p+2) /(p+1)}, \\
& E_{1}=\left(\frac{1}{2}-\frac{1}{2(p+2)}\right) \alpha_{*}^{2}
\end{aligned}
$$

where $\eta_{1}$ is the optimal constant in (24).

Our first result is concerned with the blow-up for certain local solutions with nonpositive initial energy as well as positive initial energy.

Theorem 5. Assume that (A1)-(A2), (A4), and (20) hold. Let $(u, v)$ be the unique local solution to system (1). For any fixed $\delta<1$, assuming that we can choose $\left(u_{0}, v_{0}\right),\left(u_{1}, v_{1}\right)$ satisfy

$$
E(0)<\delta E_{1}, \quad\left(l_{1}\left\|\nabla u_{0}\right\|_{2}^{2}+l_{2}\left\|\nabla v_{0}\right\|_{2}^{2}\right)^{1 / 2}>\alpha_{*} .
$$

Suppose further that

$$
\begin{aligned}
& \int_{0}^{\infty} g_{i}(s) \mathrm{d} s \\
& \leq \frac{2(p+1) m_{0}}{2(p+1)+1 /\left\{2\left[(1-\widehat{\delta})^{2}(p+2)+\widehat{\delta}(1-\widehat{\delta})\right]\right\}} \\
& \quad(i=1,2),
\end{aligned}
$$

where $\widehat{\delta}=\max \{0, \delta\}$, then $T<\infty$.

Our second result shows that certain local solutions with arbitrarily positive initial energy can also blow up.

Theorem 6. Suppose that (A1)-(A2), (A4), (20), and

$$
\int_{0}^{\infty} g_{i}(s) \mathrm{d} s \leq \frac{2(p+1) m_{0}}{2(p+1)+1 / 2(p+2)}, \quad(i=1,2)
$$


hold. Assume further that $u_{0}, v_{0} \in H_{0}^{1}(\Omega) \cap H^{2}(\Omega)$ and $u_{1}, v_{1} \in$ $L^{2}(\Omega)$ and satisfy

$0<E(0)$

$$
\begin{aligned}
<\min & \left\{\frac{\left(\int_{\Omega} u_{0} u_{1} \mathrm{~d} x+\int_{\Omega} v_{0} v_{1} \mathrm{~d} x\right)^{2}}{2\left[\left\|u_{0}\right\|_{2}^{2}+\left\|v_{0}\right\|_{2}^{2}+T_{1}\left(\left\|\nabla u_{0}\right\|_{2}^{2}+\left\|\nabla v_{0}\right\|_{2}^{2}\right)\right]},\right. \\
& \frac{\sqrt{p+3}}{(p+2)(\sqrt{p+3}-\sqrt{p+1})} \int_{\Omega}\left(u_{0} u_{1}+v_{0} v_{1}\right) \mathrm{d} x \\
& \left.-\frac{p+3}{2(p+2)}\left(\left\|u_{0}\right\|_{2}^{2}+\left\|v_{0}\right\|_{2}^{2}+\left\|\nabla u_{0}\right\|_{2}^{2}+\left\|\nabla v_{0}\right\|_{2}^{2}\right)\right\},
\end{aligned}
$$

then the solution of problem (1) blows up at a finite time $T^{*}$ in the sense of (119) below. Moreover, the upper bounds for $T^{*}$ can be estimated by

$$
T^{*} \leq 2^{(3 p+5) / 2(p+1)} \frac{(p+1) c}{2 \sqrt{a_{1}}}\left\{1-[1+\operatorname{ch}(0)]^{-1 /(p+1)}\right\},
$$

where $h(0)=\left[\left\|u_{0}\right\|_{2}^{2}+\left\|v_{0}\right\|_{2}^{2}+T_{1}\left(\left\|\nabla u_{0}\right\|_{2}^{2}+\left\|\nabla v_{0}\right\|_{2}^{2}\right)\right]^{-(p+1) / 2}$ and $a_{1}$ is given in (116) below.

Finally, we state the general decay result. For convenience, we choose especially $M(s)=m_{0}+m_{1} s^{\gamma}, m_{0}>0, m_{1} \geq 0$, and $\gamma \geq 0$. Then the energy functional $E(t)$, defined by (27), becomes

$$
\begin{aligned}
E(t):= & E(u(t), v(t)) \\
= & \frac{1}{2}\left(\left\|u_{t}\right\|_{2}^{2}+\left\|v_{t}\right\|_{2}^{2}\right) \\
+ & \frac{1}{2}\left[\left(m_{0}-\int_{0}^{t} g_{1}(s) \mathrm{d} s\right)\|\nabla u\|_{2}^{2}\right. \\
& \left.+\left(m_{0}-\int_{0}^{t} g_{2}(s) \mathrm{d} s\right)\|\nabla v\|_{2}^{2}\right] \\
+ & \frac{1}{2}\left[\left(g_{1} \diamond \nabla u\right)(t)+\left(g_{2} \diamond \nabla v\right)(t)\right. \\
& \left.+\frac{m_{1}}{\gamma+1}\left(\|\nabla u\|_{2}^{2(\gamma+1)}+\|\nabla v\|_{2}^{2(\gamma+1)}\right)\right] \\
- & \int_{\Omega} F(u, v) \mathrm{d} x .
\end{aligned}
$$

Theorem 7. Suppose that (20), (A1)-(A2), and (A3) hold, and that $\left(u_{0}, u_{1}\right) \in\left(H_{0}^{1}(\Omega) \cap H^{2}(\Omega)\right) \times L^{2}(\Omega)$ and $\left(v_{0}, v_{1}\right) \in$ $\left(H_{0}^{1}(\Omega) \cap H^{2}(\Omega)\right) \times L^{2}(\Omega)$ and satisfy $E(0)<E_{1}$ and

$$
\left(l_{1}\left\|\nabla u_{0}\right\|_{2}^{2}+l_{2}\left\|\nabla v_{0}\right\|_{2}^{2}\right)^{1 / 2}<\alpha_{*} .
$$

Then for each $t_{0}>0$, there exist two positive constants $K$ and $k$ such that the energy of (1) satisfies

$$
E(t) \leq K e^{-k \int_{t_{0}}^{t} \xi(s) \mathrm{d} s}, \quad t \geq t_{0},
$$

where $\xi(t):=\min \left\{\xi_{1}(t), \xi_{2}(t)\right\}$.
To achieve general decay result we will use a Lyapunov type technique for some perturbation energy following the method introduced in [42]. This result improves the one in $\mathrm{Li}$ et al. [23] in which only the exponential decay rates are considered.

\section{Blow-Up of Solutions with Initial Data in the Unstable Set}

In this section, we prove a finite time blow-up result for initial data in the unstable set. We need the following lemmas.

Lemma 8. Suppose that (20), (A1), (A2), and (A4) hold. Let $(u, v)$ be the solution of system (1). Assume further that $E(0)<$ $E_{1}$ and

$$
\left(l_{1}\left\|\nabla u_{0}\right\|_{2}^{2}+l_{2}\left\|\nabla v_{0}\right\|_{2}^{2}\right)^{1 / 2}>\alpha_{*} .
$$

Then there exists a constant $\alpha_{3}>\alpha_{*}$ such that

$$
\left(l_{1}\|\nabla u(t)\|_{2}^{2}+l_{2}\|\nabla v(t)\|_{2}^{2}\right)^{1 / 2} \geq \alpha_{3}, \quad \text { for } t \in[0, T) .
$$

Proof. We first note that, by (27), (24) and the definition of $B_{1}$, we have

$$
\begin{aligned}
E(t) \geq & \frac{1}{2}\left(m_{0}-\int_{0}^{t} g_{1}(s) \mathrm{d} s\right)\|\nabla u(t)\|_{2}^{2} \\
& +\frac{1}{2}\left(m_{0}-\int_{0}^{t} g_{2}(s) \mathrm{d} s\right)\|\nabla v(t)\|_{2}^{2} \\
& -\frac{1}{2(p+2)}\left(a\|u+v\|_{2(p+2)}^{2(p+2)}+2 b\|u v\|_{p+2}^{p+2}\right) \\
\geq & \frac{1}{2}\left[l_{1}\|\nabla u(t)\|_{2}^{2}+l_{2}\|\nabla v(t)\|_{2}^{2}\right] \\
& -\frac{1}{2(p+2)}\left(a\|u+v\|_{2(p+2)}^{2(p+2)}+2 b\|u v\|_{p+2}^{p+2}\right) \\
\geq & \frac{1}{2}\left[l_{1}\|\nabla u(t)\|_{2}^{2}+l_{2}\|\nabla v(t)\|_{2}^{2}\right] \\
& -\frac{B_{1}^{2(p+2)}}{2(p+2)}\left(l_{1}\|\nabla u(t)\|_{2}^{2}+l_{2}\|\nabla v(t)\|_{2}^{2}\right)^{p+2} \\
= & \frac{1}{2} \alpha^{2}-\frac{B_{1}^{2(p+2)}}{2(p+2)} \alpha^{2(p+2)}:=G(\alpha),
\end{aligned}
$$

where we have used $\bar{M}(s)=\int_{0}^{s} M(\tau) \mathrm{d} \tau \geq m_{0} s \geq 0$ and

$$
\alpha=\left(l_{1}\|\nabla u(t)\|_{2}^{2}+l_{2}\|\nabla v(t)\|_{2}^{2}\right)^{1 / 2} .
$$

It is easy to verify that $G(\alpha)$ is increasing in $\left(0, \alpha_{*}\right)$, decreasing in $\left(\alpha_{*}, \infty\right)$, and that $G(\alpha) \rightarrow-\infty$, as $\alpha \rightarrow \infty$, and

$$
G(\alpha)_{\max }=G\left(\alpha_{*}\right)=\frac{1}{2} \alpha_{*}^{2}-\frac{B_{1}^{2(p+2)}}{2(p+2)} \alpha_{*}^{2(p+2)}=E_{1},
$$


where $\alpha_{*}$ is given in (30). Since $E(0)<E_{1}$, there exists $\alpha_{3}>$ $\alpha_{*}$ such that $G\left(\alpha_{3}\right)=E(0)$. Set $\alpha_{0}=\left(l_{1}\left\|\nabla u_{0}\right\|_{2}^{2}+l_{2}\left\|\nabla v_{0}\right\|_{2}^{2}\right)^{1 / 2}$, then from (41) we can get $G\left(\alpha_{0}\right) \leq E(0)=G\left(\alpha_{3}\right)$, which implies that $\alpha_{0} \geq \alpha_{3}$.

Now we establish (40) by contradiction. First we assume that (40) is not true over $[0, T)$, then there exists $t_{0} \in(0, T)$ such that

$$
\left(l_{1}\left\|\nabla u\left(t_{0}\right)\right\|_{2}^{2}+l_{2}\left\|\nabla v\left(t_{0}\right)\right\|_{2}^{2}\right)^{1 / 2}<\alpha_{3} .
$$

By the continuity of $l_{1}\|\nabla u(t)\|_{2}^{2}+l_{2}\|\nabla v(t)\|_{2}^{2}$ we can choose $t_{0}$ such that

$$
\left(l_{1}\left\|\nabla u\left(t_{0}\right)\right\|_{2}^{2}+l_{2}\left\|\nabla v\left(t_{0}\right)\right\|_{2}^{2}\right)^{1 / 2}>\alpha_{*} .
$$

Again, the use of (41) leads to

$$
\begin{aligned}
E\left(t_{0}\right) & \geq G\left[\left(l_{1}\left\|\nabla u\left(t_{0}\right)\right\|_{2}^{2}+l_{2}\left\|\nabla u\left(t_{0}\right)\right\|_{2}^{2}\right)^{1 / 2}\right] \\
& >G\left(\alpha_{3}\right)=E(0) .
\end{aligned}
$$

This is impossible since $E(t) \leq E(0)$ for all $t \in[0, T)$.

Hence (40) is established.

Lemma 9 (see $[43,44])$. Let $L(t)$ be a positive, twice differentiable function, which satisfies, for $t>0$, the inequality

$$
L(t) L^{\prime \prime}(t)-(1+\sigma) L^{\prime}(t)^{2} \geq 0
$$

for some $\sigma>0$. If $L(0)>0$ and $L^{\prime}(0)>0$, then there exists a time $T_{*} \leq L(0) /\left[\sigma L^{\prime}(0)\right]$ such that $\lim _{t \rightarrow T_{*}^{*}} L(t)=\infty$.

Proof of Theorem 5. Assume by contradiction that the solution $(u, v)$ is global. Then we consider $L(t):[0, T] \rightarrow \mathbb{R}^{+}$ defined by

$$
\begin{aligned}
L(t)= & \|u(t)\|_{2}^{2}+\|v(t)\|_{2}^{2}+\int_{0}^{t}\|\nabla u(\tau)\|_{2}^{2} \mathrm{~d} \tau+\int_{0}^{t}\|\nabla v(\tau)\|_{2}^{2} \mathrm{~d} \tau \\
& +(T-t)\left(\left\|\nabla u_{0}\right\|_{2}^{2}+\left\|\nabla v_{0}\right\|_{2}^{2}\right)+\beta\left(t+T_{0}\right)^{2},
\end{aligned}
$$

where $T, \beta$, and $T_{0}$ are positive constants to be chosen later. Then $L(t)>0$ for all $t \in[0, T]$. Furthermore

$$
\begin{aligned}
L^{\prime}(t)= & 2 \int_{\Omega} u(t) u_{t}(t) \mathrm{d} x+2 \int_{\Omega} v(t) v_{t}(t) \mathrm{d} x \\
& +\|\nabla u(t)\|_{2}^{2}+\|\nabla v(t)\|_{2}^{2}-\left(\left\|\nabla u_{0}\right\|_{2}^{2}+\left\|\nabla v_{0}\right\|_{2}^{2}\right) \\
& +2 \beta\left(t+T_{0}\right) \\
= & 2 \int_{\Omega} u(t) u_{t}(t) \mathrm{d} x+2 \int_{\Omega} v(t) v_{t}(t) \mathrm{d} x \\
& +2 \int_{0}^{t}\left(\nabla u(\tau), \nabla u_{t}(\tau)\right) \mathrm{d} \tau \\
& +2 \int_{0}^{t}\left(\nabla v(\tau), \nabla v_{t}(\tau)\right) \mathrm{d} \tau+2 \beta\left(t+T_{0}\right),
\end{aligned}
$$

and, consequently,

$$
\begin{aligned}
L^{\prime \prime}(t)= & 2 \int_{\Omega} u(t) u_{t t}(t) \mathrm{d} x+2 \int_{\Omega} v(t) v_{t t}(t) \mathrm{d} x \\
& +2\left\|u_{t}(t)\right\|_{2}^{2}+2\left\|v_{t}(t)\right\|_{2}^{2}+2\left(\nabla u(t), \nabla u_{t}(t)\right) \\
& +2\left(\nabla v(t), \nabla v_{t}(t)\right)+2 \beta
\end{aligned}
$$

for almost every $t \in[0, T]$. Testing the first equation of system (1) with $u$ and the second equation of system (1) with $v$, integrating the results over $\Omega$, using integration by parts, and summing up, we have

$$
\begin{aligned}
\left(u_{t t}(t),\right. & u(t))+\left(v_{t t}(t), v(t)\right)+\left(\nabla u(t), \nabla u_{t}(t)\right) \\
& +\left(\nabla v(t), \nabla v_{t}(t)\right) \\
= & -M\left(\|\nabla u(t)\|_{2}^{2}\right)\|\nabla u(t)\|_{2}^{2}-M\left(\|\nabla v(t)\|_{2}^{2}\right)\|\nabla v(t)\|_{2}^{2} \\
& -\int_{\Omega} \int_{0}^{t} g_{1}(t-s) \Delta u(s) u(t) \mathrm{d} s \mathrm{~d} x \\
& -\int_{\Omega} \int_{0}^{t} g_{2}(t-s) \Delta v(s) v(t) \mathrm{d} s \mathrm{~d} x \\
& +2(p+2) \int_{\Omega} F(u, v) \mathrm{d} x,
\end{aligned}
$$

which implies

$$
\begin{aligned}
L^{\prime \prime}(t)= & 2\left\|u_{t}(t)\right\|_{2}^{2}+2\left\|v_{t}(t)\right\|_{2}^{2}-2 M\left(\|\nabla u(t)\|_{2}^{2}\right)\|\nabla u(t)\|_{2}^{2} \\
& -2 M\left(\|\nabla v(t)\|_{2}^{2}\right)\|\nabla v(t)\|_{2}^{2}+2 \beta \\
& -2 \int_{\Omega} \int_{0}^{t} g_{1}(t-s) \Delta u(s) u(t) \mathrm{d} s \mathrm{~d} x \\
& -2 \int_{\Omega} \int_{0}^{t} g_{2}(t-s) \Delta v(s) v(t) \mathrm{d} s \mathrm{~d} x \\
& +4(p+2) \int_{\Omega} F(u, v) \mathrm{d} x .
\end{aligned}
$$

Therefore, we have

$$
\begin{aligned}
L(t) L^{\prime \prime}(t)- & \frac{p+3}{2} L^{\prime}(t)^{2} \\
=2 L(t)\left(\left\|u_{t}(t)\right\|_{2}^{2}+\left\|v_{t}(t)\right\|_{2}^{2}\right. & -M\left(\|\nabla u(t)\|_{2}^{2}\right)\|\nabla u(t)\|_{2}^{2} \\
& \left.-M\left(\|\nabla v(t)\|_{2}^{2}\right)\|\nabla v(t)\|_{2}^{2}+\beta\right)
\end{aligned}
$$




$$
\begin{gathered}
-2 L(t)\left(\int_{\Omega} \int_{0}^{t} g_{1}(t-s) \Delta u(s) u(t) \mathrm{d} s \mathrm{~d} x\right. \\
+\int_{\Omega} \int_{0}^{t} g_{2}(t-s) \Delta v(s) v(t) \mathrm{d} s \mathrm{~d} x \\
\left.-2(p+2) \int_{\Omega} F(u, v) \mathrm{d} x\right)-2(p+3) \\
\times\left[\int_{\Omega} u(t) u_{t}(t) \mathrm{d} x+\int_{\Omega} v(t) v_{t}(t) \mathrm{d} x+\beta\left(t+T_{0}\right)\right. \\
+\int_{0}^{t}\left(\nabla u(\tau), \nabla u_{t}(\tau)\right) \mathrm{d} \tau \\
\left.+\int_{0}^{t}\left(\nabla v(\tau), \nabla v_{t}(\tau)\right) \mathrm{d} \tau\right]^{2} \\
=2 L(t)\left(\left\|u_{t}(t)\right\|_{2}^{2}+\left\|v_{t}(t)\right\|_{2}^{2}-M\left(\|\nabla u(t)\|_{2}^{2}\right)\|\nabla u(t)\|_{2}^{2}\right. \\
\left.-M\left(\|\nabla v(t)\|_{2}^{2}\right)\|\nabla v(t)\|_{2}^{2}+\beta\right) \\
-2 L(t)\left(\int_{\Omega}^{t} \int_{0}^{t} g_{1}(t-s) \Delta u(s) u(t) \mathrm{d} s \mathrm{~d} x\right. \\
+\int_{\Omega} \int_{0}^{t} g_{2}(t-s) \Delta v(s) v(t) \mathrm{d} s \mathrm{~d} x \\
\left.-2(p+2) \int_{\Omega} F(u, v) \mathrm{d} x\right) \\
+2(p+3)\{H(t) \\
-\left[L(t)-(T-t)\left(\left\|\nabla u_{0}\right\|_{2}^{2}+\left\|\nabla v_{0}\right\|_{2}^{2}\right)\right] \\
\times \Psi(t)\},
\end{gathered}
$$

where $\Psi(t), H(t):[0, T] \rightarrow \mathbb{R}^{+}$are the functions defined by

$$
\begin{gathered}
\Psi(t)=\left\|u_{t}(t)\right\|_{2}^{2}+\left\|v_{t}(t)\right\|_{2}^{2} \\
+\int_{0}^{t}\left\|\nabla u_{t}(\tau)\right\|_{2}^{2} \mathrm{~d} \tau+\int_{0}^{t}\left\|\nabla v_{t}(\tau)\right\|_{2}^{2} \mathrm{~d} \tau+\beta, \\
H(t)=\left(\|u(t)\|_{2}^{2}+\|v(t)\|_{2}^{2}+\int_{0}^{t}\|\nabla u(\tau)\|_{2}^{2} \mathrm{~d} \tau\right. \\
\left.+\int_{0}^{t}\|\nabla v(\tau)\|_{2}^{2} \mathrm{~d} \tau+\beta\left(t+T_{0}\right)^{2}\right) \Psi(t) \\
-\left[\int_{\Omega}\left[u(t) u_{t}(t)+v(t) v_{t}(t)\right] \mathrm{d} x\right. \\
\quad+\int_{0}^{t}\left[\left(\nabla u(\tau), \nabla u_{t}(\tau)\right)+\left(\nabla v(\tau), \nabla v_{t}(\tau)\right)\right] \mathrm{d} \tau \\
\left.+\beta\left(t+T_{0}\right)\right]^{2} .
\end{gathered}
$$

Using the Cauchy-Schwarz inequality, we obtain

$$
\left(\int_{\Omega} u(t) u_{t}(t) \mathrm{d} x\right)^{2} \leq\|u(t)\|_{2}^{2}\left\|u_{t}(t)\right\|_{2}^{2},
$$

(the similar inequality for $v(t)$ holds true)

$$
\left(\int_{0}^{t}\left(\nabla u(\tau), \nabla u_{t}(\tau)\right) \mathrm{d} \tau\right)^{2} \leq \int_{0}^{t}\|\nabla u(\tau)\|_{2}^{2} \mathrm{~d} \tau \int_{0}^{t}\left\|\nabla u_{t}(\tau)\right\|_{2}^{2} \mathrm{~d} \tau
$$

(the similar inequality for $v(t)$ ),

$$
\begin{aligned}
\int_{\Omega} u(t) & u_{t}(t) \mathrm{d} x \int_{\Omega} v(t) v_{t}(t) \mathrm{d} x \\
& \leq\|u(t)\|_{2}\left\|v_{t}(t)\right\|_{2}\left\|u_{t}(t)\right\|_{2}\|v(t)\|_{2} \\
& \leq \frac{1}{2}\|u(t)\|_{2}^{2}\left\|v_{t}(t)\right\|_{2}^{2}+\frac{1}{2}\left\|u_{t}(t)\right\|_{2}^{2}\|v(t)\|_{2}^{2} .
\end{aligned}
$$

Similarly, we have

$$
\begin{gathered}
\int_{\Omega} u(t) u_{t}(t) \mathrm{d} x \int_{0}^{t}\left(\nabla u(\tau), \nabla u_{t}(\tau)\right) \mathrm{d} \tau \\
\leq \frac{1}{2}\|u(t)\|_{2}^{2} \int_{0}^{t}\left\|\nabla u_{t}(\tau)\right\|_{2}^{2} \mathrm{~d} \tau \\
+\frac{1}{2}\left\|u_{t}(t)\right\|_{2}^{2} \int_{0}^{t}\|\nabla u(\tau)\|_{2}^{2} \mathrm{~d} \tau,
\end{gathered}
$$

(the similar inequality for $v(t)$ holds true)

$$
\begin{aligned}
\int_{\Omega} u(t) & u_{t}(t) \mathrm{d} x \int_{0}^{t}\left(\nabla v(\tau), \nabla v_{t}(\tau)\right) \mathrm{d} \tau \\
\leq & \frac{1}{2}\|u(t)\|_{2}^{2} \int_{0}^{t}\left\|\nabla v_{t}(\tau)\right\|_{2}^{2} \mathrm{~d} \tau \\
& +\frac{1}{2}\left\|u_{t}(t)\right\|_{2}^{2} \int_{0}^{t}\|\nabla v(\tau)\|_{2}^{2} \mathrm{~d} \tau, \\
\int_{\Omega} v(t) & v_{t}(t) \mathrm{d} x \int_{0}^{t}\left(\nabla u(\tau), \nabla u_{t}(\tau)\right) \mathrm{d} \tau \\
\leq & \frac{1}{2}\|v(t)\|_{2}^{2} \int_{0}^{t}\left\|\nabla u_{t}(\tau)\right\|_{2}^{2} \mathrm{~d} \tau \\
& +\frac{1}{2}\left\|v_{t}(t)\right\|_{2}^{2} \int_{0}^{t}\|\nabla u(\tau)\|_{2}^{2} \mathrm{~d} \tau, \\
\int_{0}^{t}(\nabla u & \left.(\tau), \nabla u_{t}(\tau)\right) \mathrm{d} \tau \int_{0}^{t}\left(\nabla v(\tau), \nabla v_{t}(\tau)\right) \mathrm{d} \tau \\
\leq & \frac{1}{2} \int_{0}^{t}\|\nabla u(\tau)\|_{2}^{2} \mathrm{~d} \tau \int_{0}^{t}\left\|\nabla v_{t}(\tau)\right\|_{2}^{2} \mathrm{~d} \tau \\
& +\frac{1}{2} \int_{0}^{t}\left\|\nabla u_{t}(\tau)\right\|_{2}^{2} \mathrm{~d} \tau \int_{0}^{t}\|\nabla v(\tau)\|_{2}^{2} \mathrm{~d} \tau
\end{aligned}
$$


By Hölder's inequality and Young's inequality, we obtain

$$
\begin{aligned}
\beta(t+ & \left.T_{0}\right) \int_{\Omega} u(t) u_{t}(t) \mathrm{d} x \\
& \leq \beta\left(t+T_{0}\right)\|u(t)\|_{2}\left\|u_{t}(t)\right\|_{2} \\
& \leq \frac{1}{2} \beta\|u(t)\|_{2}^{2}+\frac{1}{2} \beta\left(t+T_{0}\right)^{2}\left\|u_{t}(t)\right\|_{2}^{2} .
\end{aligned}
$$

(the similar inequality for $v(t)$ holds true)

$$
\begin{aligned}
\beta(t+ & \left.T_{0}\right) \int_{0}^{t}\left(\nabla u(\tau), \nabla u_{t}(\tau)\right) \mathrm{d} \tau \\
& \leq \beta\left(t+T_{0}\right) \int_{0}^{t}\|\nabla u(\tau)\|_{2}\left\|\nabla u_{t}(\tau)\right\|_{2} \mathrm{~d} \tau \\
& \leq \frac{1}{2} \beta \int_{0}^{t}\|\nabla u(\tau)\|_{2}^{2} \mathrm{~d} \tau+\frac{1}{2} \beta\left(t+T_{0}\right)^{2} \int_{0}^{t}\left\|\nabla u_{t}(\tau)\right\|_{2}^{2} \mathrm{~d} \tau .
\end{aligned}
$$

(the similar inequality for $v(t)$ holds true).

The previous inequalities imply that $H(t) \geq 0$ for every $t \epsilon$ $[0, T]$. Using (53), we get

$$
L(t) L^{\prime \prime}(t)-\frac{p+3}{2} L^{\prime}(t)^{2} \geq L(t) \Phi(t)
$$

for almost every $t \in[0, T]$, where $\Phi(t):[0, T] \rightarrow \mathbb{R}^{+}$is the map defined by

$$
\begin{aligned}
\Phi(t)= & -2(p+2)\left(\left\|u_{t}(t)\right\|_{2}^{2}+\left\|v_{t}(t)\right\|_{2}^{2}\right) \\
& -2 M\left(\|\nabla u(t)\|_{2}^{2}\right)\|\nabla u(t)\|_{2}^{2} \\
& -2 M\left(\|\nabla v(t)\|_{2}^{2}\right)\|\nabla v(t)\|_{2}^{2} \\
& -2\left(\int_{\Omega} \int_{0}^{t} g_{1}(t-s) \Delta u(s) u(t) \mathrm{d} s \mathrm{~d} x\right. \\
& \left.\quad+\int_{\Omega} \int_{0}^{t} g_{2}(t-s) \Delta v(s) v(t) \mathrm{d} s \mathrm{~d} x\right) \\
& -2(p+2) \beta \\
& -2(p+3)\left(\int_{0}^{t}\left\|\nabla u_{t}(\tau)\right\|_{2}^{2} \mathrm{~d} \tau+\int_{0}^{t}\left\|\nabla v_{t}(\tau)\right\|_{2}^{2} \mathrm{~d} \tau\right) \\
& +4(p+2) \int_{\Omega} F(u, v) \mathrm{d} x .
\end{aligned}
$$

For the fourth term on the right hand side of (59), we have

$$
\begin{array}{r}
-\int_{\Omega} \int_{0}^{t} g_{1}(t-s) \Delta u(s) u(t) \mathrm{d} s \mathrm{~d} x \\
=\int_{0}^{t} g_{1}(t-s) \int_{\Omega} \nabla u(s) \cdot \nabla u(t) \mathrm{d} x \mathrm{~d} s
\end{array}
$$

$$
\begin{aligned}
= & \int_{0}^{t} g_{1}(t-s) \int_{\Omega} \nabla u(t) \cdot(\nabla u(s)-\nabla u(t)) \mathrm{d} x \mathrm{~d} s \\
& +\left(\int_{0}^{t} g_{1}(s) \mathrm{d} s\right)\|\nabla u(t)\|_{2}^{2} .
\end{aligned}
$$

Similarly,

$$
\begin{aligned}
& -\int_{\Omega} \int_{0}^{t} g_{2}(t-s) \Delta v(s) v(t) \mathrm{d} s \mathrm{~d} x \\
& =\int_{0}^{t} g_{2}(t-s) \int_{\Omega} \nabla v(t) \cdot(\nabla v(s)-\nabla v(t)) \mathrm{d} x \mathrm{~d} s \\
& \quad+\left(\int_{0}^{t} g_{2}(s) \mathrm{d} s\right)\|\nabla v(t)\|_{2}^{2} .
\end{aligned}
$$

Combining (59), (60) with (61), we get

$$
\begin{aligned}
\Phi(t)= & -2(p+2)\left(\left\|u_{t}(t)\right\|_{2}^{2}+\left\|v_{t}(t)\right\|_{2}^{2}\right) \\
& -2 M\left(\|\nabla u(t)\|_{2}^{2}\right)\|\nabla u(t)\|_{2}^{2} \\
& -2 M\left(\|\nabla v(t)\|_{2}^{2}\right)\|\nabla v(t)\|_{2}^{2} \\
& +2 \int_{0}^{t} g_{1}(s) \mathrm{d} s\|\nabla u(t)\|_{2}^{2}+2 \int_{0}^{t} g_{2}(s) \mathrm{d}\|\nabla v(t)\|_{2}^{2} \\
& +4(p+2) \int_{\Omega} F(u, v) \mathrm{d} x-2(p+2) \beta \\
& +2 \int_{0}^{t} g_{1}(t-s) \int_{\Omega} \nabla u(t)(\nabla u(s)-\nabla u(t)) \mathrm{d} x \mathrm{~d} s \\
& -2(p+3) \int_{0}^{t}\left\|\nabla u_{t}(\tau)\right\|_{2}^{2} \mathrm{~d} \tau \\
& +2 \int_{0}^{t} g_{2}(t-s) \int_{\Omega} \nabla v(t)(\nabla v(s)-\nabla v(t)) \mathrm{d} x \mathrm{~d} s \\
& -2(p+3) \int_{0}^{t}\left\|\nabla v_{t}(\tau)\right\|_{2}^{2} \mathrm{~d} \tau .
\end{aligned}
$$

Since, we have

$2 \int_{0}^{t} g_{i}(t-s) \int_{\Omega} \nabla u(t) \cdot(\nabla u(s)-\nabla u(t)) \mathrm{d} x \mathrm{~d} s$

$$
\geq-\varepsilon \int_{0}^{t} g_{i}(t-s)\|\nabla u(s)-\nabla u(t)\|_{2}^{2} \mathrm{~d} s
$$

$$
\begin{aligned}
& -\frac{1}{\varepsilon}\left(\int_{0}^{t} g_{i}(s) \mathrm{d} s\right)\|\nabla u(t)\|_{2}^{2} \\
= & -\varepsilon\left(g_{i} \diamond \nabla u\right)(t)-\frac{1}{\varepsilon}\left(\int_{0}^{t} g_{i}(s) \mathrm{d} s\right)\|\nabla u(t)\|_{2}^{2}, \quad i=1,2,
\end{aligned}
$$


for any $\varepsilon>0$, inserting (63) into (62) and utilizing (27), we have

$$
\begin{aligned}
& \Phi(t) \geq-2(p+2)\left(\left\|u_{t}(t)\right\|_{2}^{2}+\left\|v_{t}(t)\right\|_{2}^{2}\right) \\
& -\varepsilon\left[\left(g_{1} \diamond \nabla u\right)(t)+\left(g_{2} \diamond \nabla v\right)(t)\right] \\
& +4(p+2) \int_{\Omega} F(u, v) \mathrm{d} x \\
& +\left(2-\frac{1}{\varepsilon}\right) \\
& \times\left[\int_{0}^{t} g_{1}(s) \mathrm{d} s\|\nabla u(t)\|_{2}^{2}+\int_{0}^{t} g_{2}(s) \mathrm{d} s\|\nabla v(t)\|_{2}^{2}\right] \\
& -2(p+3)\left(\int_{0}^{t}\left\|\nabla u_{t}(\tau)\right\|_{2}^{2} \mathrm{~d} \tau+\int_{0}^{t}\left\|\nabla v_{t}(\tau)\right\|_{2}^{2} \mathrm{~d} \tau\right) \\
& -2 M\left(\|\nabla u(t)\|_{2}^{2}\right)\|\nabla u(t)\|_{2}^{2} \\
& -2 M\left(\|\nabla v(t)\|_{2}^{2}\right)\|\nabla v(t)\|_{2}^{2} \\
& -2(p+2) \beta-4(p+2) E(t)+4(p+2) E(t) \\
& =-4(p+2) E(t)+2(p+2) \bar{M}\left(\|\nabla u(t)\|_{2}^{2}\right) \\
& -2 M\left(\|\nabla u(t)\|_{2}^{2}\right)\|\nabla u(t)\|_{2}^{2} \\
& +2(p+2) \bar{M}\left(\|\nabla v(t)\|_{2}^{2}\right) \\
& -2 M\left(\|\nabla v(t)\|_{2}^{2}\right)\|\nabla v(t)\|_{2}^{2} \\
& -2\left(p+1+\frac{1}{2 \varepsilon}\right) \int_{0}^{t} g_{1}(s) \mathrm{d} s\|\nabla u(t)\|_{2}^{2} \\
& -2(p+2) \beta \\
& -2\left(p+1+\frac{1}{2 \varepsilon}\right) \int_{0}^{t} g_{2}(s) \mathrm{d} s\|\nabla v(t)\|_{2}^{2} \\
& -2(p+3) \int_{0}^{t}\left(\left\|\nabla u_{t}(\tau)\right\|_{2}^{2}+\left\|\nabla v_{t}(\tau)\right\|_{2}^{2}\right) \mathrm{d} \tau \\
& +[2(p+2)-\varepsilon]\left[\left(g_{1} \diamond \nabla u\right)(t)+\left(g_{2} \diamond \nabla v\right)(t)\right] .
\end{aligned}
$$

Using (29) and (A4), we have

$$
\begin{aligned}
\Phi(t) \geq & -4(p+2) E(0)+2(p+1) \\
& \times\left(m_{0}-\int_{0}^{t} g_{1}(s) \mathrm{d} s\right)\|\nabla u(t)\|_{2}^{2} \\
& -\frac{1}{\varepsilon}\left(\int_{0}^{t} g_{1}(s) \mathrm{d} s\right)\|\nabla u(t)\|_{2}^{2} \\
& +2(p+1)\left(m_{0}-\int_{0}^{t} g_{2}(s) \mathrm{d} s\right)\|\nabla v(t)\|_{2}^{2} \\
& -\frac{1}{\varepsilon}\left(\int_{0}^{t} g_{2}(s) \mathrm{d} s\right)\|\nabla v(t)\|_{2}^{2}-2(p+2) \beta
\end{aligned}
$$

$$
\begin{aligned}
& +2(p+1) \int_{0}^{t}\left(\left\|\nabla u_{t}(\tau)\right\|_{2}^{2}+\left\|\nabla v_{t}(\tau)\right\|_{2}^{2}\right) \mathrm{d} \tau \\
& +[2(p+2)-\varepsilon]\left[\left(g_{1} \diamond \nabla u\right)(t)+\left(g_{2} \diamond \nabla v\right)(t)\right] \\
= & -4(p+2) E(0) \\
+ & {\left[2(p+1) m_{0}-\left(2(p+1)+\frac{1}{\varepsilon}\right) \int_{0}^{t} g_{1}(s) \mathrm{d} s\right] } \\
& \times\|\nabla u(t)\|_{2}^{2}-2(p+2) \beta \\
& +\left[2(p+1) m_{0}-\left(2(p+1)+\frac{1}{\varepsilon}\right) \int_{0}^{t} g_{2}(s) \mathrm{d} s\right] \\
& \times\|\nabla v(t)\|_{2}^{2} \\
& +[2(p+2)-\varepsilon]\left[\left(g_{1} \diamond \nabla u\right)(t)+\left(g_{2} \diamond \nabla v\right)(t)\right] \\
& +2(p+1) \int_{0}^{t}\left(\left\|\nabla u_{t}(\tau)\right\|_{2}^{2}+\left\|\nabla v_{t}(\tau)\right\|_{2}^{2}\right) \mathrm{d} \tau .
\end{aligned}
$$

If $\delta<0$, that is, $E(0)<0$, we choose $\varepsilon=2(p+2)$ in (65) and $\beta$ small enough such that $\beta \leq-2 E(0)$. Then by (32), we have

$$
\begin{aligned}
\Phi(t) \geq & {\left[2(p+1) m_{0}\right.} \\
& \left.\quad-\left(2(p+1)+\frac{1}{2(p+2)}\right) \int_{0}^{t} g_{1}(s) \mathrm{d} s\right]
\end{aligned}
$$$$
\times\|\nabla u(t)\|_{2}^{2}
$$$$
+\left[2(p+1) m_{0}\right.
$$$$
\left.-\left(2(p+1)+\frac{1}{2(p+2)}\right) \int_{0}^{t} g_{2}(s) \mathrm{d} s\right]
$$

$\times\|\nabla v(t)\|_{2}^{2}$

$$
+2(p+1) \int_{0}^{t}\left(\left\|\nabla u_{t}(\tau)\right\|_{2}^{2}+\left\|\nabla v_{t}(\tau)\right\|_{2}^{2}\right) \mathrm{d} \tau
$$$$
+2(p+2)(-2 E(0)-\beta) \geq 0 \text {. }
$$

If $0 \leq \delta<1$, that is, $0 \leq E(0)<\delta E_{1}<E_{1}$, we choose $\varepsilon=2(p+2)(1-\delta)+2 \delta$ and $\beta=2\left(\delta E_{1}-E(0)\right)$ in (65). Then, we get

$\Phi(t)$

$$
\begin{aligned}
& \geq-4(p+2) \delta E_{1} \\
& \quad+\left[2(p+1) m_{0}-\left(2(p+1)+\frac{1}{2(p+2)(1-\delta)+2 \delta}\right)\right. \\
& \left.\quad \times \int_{0}^{t} g_{1}(s) \mathrm{d} s\right] \\
& \quad \times\|\nabla u(t)\|_{2}^{2}
\end{aligned}
$$




$$
\begin{aligned}
& +\left[2(p+1) m_{0}-\left(2(p+1)+\frac{1}{2(p+2)(1-\delta)+2 \delta}\right)\right. \\
& \left.\quad \times \int_{0}^{t} g_{2}(s) \mathrm{d} s\right] \\
& \times\|\nabla v(t)\|_{2}^{2} \\
& +2(p+1) \int_{0}^{t}\left(\left\|\nabla u_{t}(\tau)\right\|_{2}^{2}+\left\|\nabla v_{t}(\tau)\right\|_{2}^{2}\right) \mathrm{d} \tau \\
& +2(p+1) \delta\left[\left(g_{1} \diamond \nabla u\right)(t)+\left(g_{2} \diamond \nabla v\right)(t)\right] .
\end{aligned}
$$

By (32), we have (notice that $\widehat{\delta}=\delta$ due to $\delta \geq 0$ )

$$
\begin{aligned}
& \int_{0}^{t} g_{i}(s) \mathrm{d} s \\
& \quad \leq \int_{0}^{\infty} g_{i}(s) \mathrm{d} s \\
& \quad \leq \frac{2(p+1) m_{0}}{2(p+1)+1 /\left\{2\left[(1-\delta)^{2}(p+2)+\delta(1-\delta)\right]\right\}} \\
& \quad=\frac{2(p+1) m_{0}-2 \delta(p+1) m_{0}}{2(1-\delta)(p+1)+1 /\{2(1-\delta)(p+2)+2 \delta\}}, \\
& \quad(i=1,2) ;
\end{aligned}
$$

that is,

$$
\begin{aligned}
2 \delta(p+1) & {\left[m_{0}-\int_{0}^{t} g_{i}(s) \mathrm{d} s\right] } \\
\leq & 2(p+1) m_{0} \\
& -\left(2(p+1)+\frac{1}{2(p+2)(1-\delta)+2 \delta}\right) \int_{0}^{t} g_{i}(s) \mathrm{d} s
\end{aligned}
$$

for $i=1,2$. Therefore, from the above two inequalities and (A2), we can get

$$
\begin{aligned}
\Phi(t) \geq & -4(p+2) \delta E_{1} \\
& +2 \delta(p+1)\left(l_{1}\|\nabla u(t)\|_{2}^{2}+l_{2}\|\nabla v(t)\|_{2}^{2}\right) .
\end{aligned}
$$

Since

$$
\left(l_{1}\left\|\nabla u_{0}\right\|_{2}^{2}+l_{2}\left\|\nabla v_{0}\right\|_{2}^{2}\right)^{1 / 2}>\alpha_{*},
$$

by Lemma 8 , there exists a constant $\alpha_{3}>\alpha_{*}$ such that

$$
\left(l_{1}\|\nabla u(t)\|_{2}^{2}+l_{2}\|\nabla v(t)\|_{2}^{2}\right)^{1 / 2} \geq \alpha_{3},
$$

which implies

$$
\begin{aligned}
& \frac{p+1}{2(p+2)}\left(l_{1}\|\nabla u(t)\|_{2}^{2}+l_{2}\|\nabla v(t)\|_{2}^{2}\right) \\
& \quad \geq \frac{p+1}{2(p+2)} \alpha_{3}^{2}>\frac{p+1}{2(p+2)} \alpha_{*}^{2}>E_{1} .
\end{aligned}
$$

It follows from (67) and (73) that

$$
\Phi(t) \geq 4(p+2)\left[\delta E_{1}-\delta E_{1}\right] \geq 0 .
$$

Therefore, by (58), (66), and (74), we obtain

$$
L(t) L^{\prime \prime}(t)-\frac{p+3}{2} L^{\prime}(t)^{2} \geq 0
$$

for almost every $t \in[0, T]$. By (49), we then choose $T_{0}$ sufficiently large such that

$$
\begin{gathered}
(p+1)\left(\int_{\Omega} u_{0} u_{1} \mathrm{~d} x+\int_{\Omega} v_{0} v_{1} \mathrm{~d} x+\beta T_{0}\right) \\
>\left\|\nabla u_{0}\right\|_{2}^{2}+\left\|\nabla v_{0}\right\|_{2}^{2}>0,
\end{gathered}
$$

consequently,

$$
L^{\prime}(0)=2 \int_{\Omega} u_{0} u_{1} \mathrm{~d} x+2 \int_{\Omega} v_{0} v_{1} \mathrm{~d} x+2 \beta T_{0}>0 .
$$

Then by (76) and (77), we choose $T$ large enough so that

$$
\begin{gathered}
T>\left(\left\|u_{0}\right\|_{2}^{2}+\left\|v_{0}\right\|_{2}^{2}+\beta T_{0}^{2}\right) \\
\times\left((p+1)\left(\int_{\Omega} u_{0} u_{1} \mathrm{~d} x+\int_{\Omega} v_{0} v_{1} \mathrm{~d} x+\beta T_{0}\right)\right. \\
\left.-\left(\left\|\nabla u_{0}\right\|_{2}^{2}+\left\|\nabla v_{0}\right\|_{2}^{2}\right)\right)^{-1}>0,
\end{gathered}
$$

which ensures that $T>(2 /(p+1))\left(L(0) / L^{\prime}(0)\right)$. As $(p+3) / 2>$ 1 , letting $\sigma=(p+1) / 2$, we can select $T_{*}$ such that $T_{*} \leq$ $L(0) / \sigma L^{\prime}(0) \leq T$. By using Lemma 9 , we get $\lim _{t \rightarrow T_{*}^{-}} L(t)=$ $\infty$. This implies that

$$
\lim _{t \rightarrow T_{*}^{-}}\left(\|\nabla u(t)\|_{2}^{2}+\|\nabla v(t)\|_{2}^{2}\right)=\infty,
$$

which is a contradiction. Thus, $T<\infty$.

\section{Blow-Up of Solutions with Arbitrarily Positive Initial Energy}

In this section, we prove the second blow-up result (Theorem 6) for solutions with arbitrarily positive initial energy. In order to attain our aim, we need the following three lemmas.

Lemma 10 (see [4]). Let $\delta^{*}>0$ and $B(t) \in C^{2}(0, \infty)$ be a nonnegative function satisfying

$$
B^{\prime \prime}(t)-4\left(\delta^{*}+1\right) B^{\prime}(t)+4\left(\delta^{*}+1\right) B(t) \geq 0 .
$$

If

$$
B^{\prime}(0)>r_{2} B(0)+K_{0}
$$

then

$$
B^{\prime}(t)>K_{0}
$$

for $t>0$, where $K_{0}$ is a constant and $r_{2}=2\left(\delta^{*}+1\right)-$ $2 \sqrt{\left(\delta^{*}+1\right) \delta^{*}}$ is the smallest root of the equation

$$
r^{2}-4\left(\delta^{*}+1\right) r+4\left(\delta^{*}+1\right)=0 .
$$


Lemma 11 (see [4]). If $h(t)$ is a nonincreasing function on $[0, \infty)$ and satisfies the differential inequality

$$
h^{\prime}(t)^{2} \geq a_{*}+b_{*} h(t)^{2+1 / \delta^{*}} \quad \text { for } t \geq 0,
$$

where $a_{*}>0, \delta^{*}>0$, and $b_{*}>0$, then there exists a finite time $T^{*}$ such that $\lim _{t \rightarrow T^{*}-} h(t)=0$ and the upper bound of $T^{*}$ is estimated by

$$
T^{*} \leq 2^{\left(3 \delta^{*}+1\right) / 2 \delta^{*}} \frac{\delta^{*} c}{\sqrt{a_{*}}}\left\{1-[1+\operatorname{ch}(0)]^{-1 / 2 \delta^{*}}\right\},
$$

where $c=\left(a_{*} / b_{*}\right)^{2+1 / \delta^{*}}$.

For the next lemma, we define

$$
\begin{aligned}
K(t):= & K(u(t), v(t))=\|u(t)\|_{2}^{2}+\|v(t)\|_{2}^{2} \\
& +\int_{0}^{t}\|\nabla u(\tau)\|_{2}^{2} \mathrm{~d} \tau+\int_{0}^{t}\|\nabla v(\tau)\|_{2}^{2} \mathrm{~d} \tau, \quad t \geq 0
\end{aligned}
$$

Lemma 12. Assume that the conditions of Theorem 6 hold and let $(u, v)$ be a solution of $(1)$, then

$$
K^{\prime}(t)>\left\|\nabla u_{0}\right\|_{2}^{2}+\left\|\nabla v_{0}\right\|_{2}^{2}, \quad t>0 .
$$

Proof. By (86), we have

$$
\begin{gathered}
K^{\prime}(t)=2 \int_{\Omega} u u_{t} \mathrm{~d} x+2 \int_{\Omega} v v_{t} \mathrm{~d} x+\|\nabla u\|_{2}^{2}+\|\nabla v\|_{2}^{2}, \\
K^{\prime \prime}(t)=2\left\|u_{t}\right\|_{2}^{2}+2\left\|v_{t}\right\|_{2}^{2}+2 \int_{\Omega} u u_{t t} \mathrm{~d} x \\
+2 \int_{\Omega} v v_{t t} \mathrm{~d} x+2 \int_{\Omega} \nabla u \cdot \nabla u_{t} \mathrm{~d} x \\
+2 \int_{\Omega} \nabla v \cdot \nabla v_{t} \mathrm{~d} x
\end{gathered}
$$

Testing the first equation of system (1) with $u$ and testing the second equation of system (1) with $v$ and plugging the results into the expression of $K^{\prime \prime}(t)$ we obtain

$$
\begin{aligned}
K^{\prime \prime}(t)= & 2\left(\left\|u_{t}\right\|_{2}^{2}+\left\|v_{t}\right\|_{2}^{2}\right)-2 M\left(\|\nabla u\|_{2}^{2}\right)\|\nabla u\|_{2}^{2} \\
& -2 M\left(\|\nabla v\|_{2}^{2}\right)\|\nabla v\|_{2}^{2}+4(p+2) \int_{\Omega} F(u, v) \mathrm{d} x \\
& +2 \int_{0}^{t} \int_{\Omega} g_{1}(t-s) \nabla u(s) \cdot \nabla u(t) \mathrm{d} x \mathrm{~d} s \\
& +2 \int_{0}^{t} \int_{\Omega} g_{2}(t-s) \nabla v(s) \cdot \nabla v(t) \mathrm{d} x \mathrm{~d} s .
\end{aligned}
$$

By (27), (29), and (90), we get

$$
\begin{aligned}
K^{\prime \prime}(t)- & 2(p+3)\left(\left\|u_{t}\right\|_{2}^{2}+\left\|v_{t}\right\|_{2}^{2}\right) \\
\geq & -4(p+2) E(0) \\
& +4(p+2) \int_{0}^{t}\left(\left\|\nabla u_{t}(\tau)\right\|_{2}^{2}+\left\|\nabla v_{t}(\tau)\right\|_{2}^{2}\right) \mathrm{d} \tau \\
& +2(p+2)\left[\bar{M}\left(\|\nabla u\|_{2}^{2}\right)+\bar{M}\left(\|\nabla v\|_{2}^{2}\right)\right] \\
& -\left[2 M\left(\|\nabla u\|_{2}^{2}\right)+2(p+2) \int_{0}^{t} g_{1}(s) \mathrm{d} s\right]\|\nabla u\|_{2}^{2} \\
& -\left[2 M\left(\|\nabla v\|_{2}^{2}\right)+2(p+2) \int_{0}^{t} g_{2}(s) \mathrm{d} s\right]\|\nabla v\|_{2}^{2} \\
& +2(p+2)\left[\left(g_{1} \diamond \nabla u\right)(t)+\left(g_{2} \diamond \nabla v\right)(t)\right] \\
& -2(p+2) \int_{0}^{t}\left[\left(g_{1}^{\prime} \diamond \nabla u\right)(\tau)+\left(g_{2}^{\prime} \diamond \nabla v\right)(\tau)\right] \mathrm{d} \tau \\
& +2 \int_{0}^{t} \int_{\Omega} g_{1}(t-s) \nabla u(s) \cdot \nabla u(t) \mathrm{d} x \mathrm{~d} s \\
& +2 \int_{0}^{t} \int_{\Omega} g_{2}(t-s) \nabla v(s) \cdot \nabla v(t) \mathrm{d} x \mathrm{~d} s .
\end{aligned}
$$

By using Hölder's inequality and Young's inequality, we have

$$
\begin{aligned}
2 \int_{0}^{t} \int_{\Omega} & g_{1}(t-s) \nabla u(s) \cdot \nabla u(t) \mathrm{d} x \mathrm{~d} s \\
= & 2 \int_{0}^{t} g_{1}(t-s) \int_{\Omega} \nabla u(t)(\nabla u(s)-\nabla u(t)) \mathrm{d} x \mathrm{~d} s \\
& +2\left(\int_{0}^{t} g_{1}(s) \mathrm{d} s\right)\|\nabla u(t)\|_{2}^{2} \\
\geq & -2(p+2)\left(g_{1} \diamond \nabla u\right)(t) \\
& +\left(2-\frac{1}{2(p+2)}\right)\left(\int_{0}^{t} g_{1}(s) \mathrm{d} s\right)\|\nabla u(t)\|_{2}^{2} .
\end{aligned}
$$

Similarly,

$$
\begin{aligned}
2 \int_{0}^{t} \int_{\Omega} & g_{2}(t-s) \nabla v(s) \cdot \nabla v(t) \mathrm{d} x \mathrm{~d} s \\
\geq & -2(p+2)\left(g_{2} \diamond \nabla v\right)(t) \\
& \quad+\left(2-\frac{1}{2(p+2)}\right)\left(\int_{0}^{t} g_{2}(s) \mathrm{d} s\right)\|\nabla v(t)\|_{2}^{2} .
\end{aligned}
$$


Then taking (92) and (93) into account, we obtain

$$
\begin{aligned}
& K^{\prime \prime}(t)-2(p+3)\left(\left\|u_{t}\right\|_{2}^{2}+\left\|v_{t}\right\|_{2}^{2}\right) \\
& \geq-4(p+2) E(0)+4(p+2) \\
& \times \int_{0}^{t}\left(\left\|\nabla u_{t}(\tau)\right\|_{2}^{2}+\left\|\nabla v_{t}(\tau)\right\|_{2}^{2}\right) \mathrm{d} \tau \\
& +\left\{2(p+2) \bar{M}\left(\|\nabla u\|_{2}^{2}\right)\right. \\
& -\left[2 M\left(\|\nabla u\|_{2}^{2}\right)\right. \\
& \left.+\left(2(p+1)+\frac{1}{2(p+2)}\right) \int_{0}^{t} g_{1}(s) \mathrm{d} s\right] \\
& \left.\times\|\nabla u\|_{2}^{2}\right\} \\
& +\left\{2(p+2) \bar{M}\left(\|\nabla v\|_{2}^{2}\right)\right. \\
& -\left[2 M\left(\|\nabla v\|_{2}^{2}\right)\right. \\
& \left.+\left(2(p+1)+\frac{1}{2(p+2)}\right) \int_{0}^{t} g_{2}(s) \mathrm{d} s\right] \\
& \left.\times\|\nabla v\|_{2}^{2}\right\} \text {. }
\end{aligned}
$$

Thus, by (A4) and (33), we get

$$
\begin{aligned}
& K^{\prime \prime}(t)-2(p+3)\left(\left\|u_{t}\right\|_{2}^{2}+\left\|v_{t}\right\|_{2}^{2}\right) \\
& \geq-4(p+2) E(0)+4(p+2) \\
& \quad \times \int_{0}^{t}\left(\left\|\nabla u_{t}(\tau)\right\|_{2}^{2}+\left\|\nabla v_{t}(\tau)\right\|_{2}^{2}\right) \mathrm{d} \tau .
\end{aligned}
$$

We note that

$$
\begin{gathered}
\|\nabla u(t)\|_{2}^{2}-\left\|\nabla u_{0}\right\|_{2}^{2}=2 \int_{0}^{t} \int_{\Omega} \nabla u \cdot \nabla u_{t} \mathrm{~d} x \mathrm{~d} \tau, \\
\|\nabla v(t)\|_{2}^{2}-\left\|\nabla v_{0}\right\|_{2}^{2}=2 \int_{0}^{t} \int_{\Omega} \nabla v \cdot \nabla v_{t} \mathrm{~d} x \mathrm{~d} \tau .
\end{gathered}
$$

By Hölder's inequality and Young's inequality, we obtain from (96)

$$
\begin{gathered}
\|\nabla u(t)\|_{2}^{2}+\|\nabla v(t)\|_{2}^{2} \leq\left\|\nabla u_{0}\right\|_{2}^{2}+\left\|\nabla v_{0}\right\|_{2}^{2} \\
+\int_{0}^{t}\left(\|\nabla u(\tau)\|_{2}^{2}+\|\nabla v(\tau)\|_{2}^{2}\right) \mathrm{d} \tau \\
+\int_{0}^{t}\left(\left\|\nabla u_{t}(\tau)\right\|_{2}^{2}+\left\|\nabla v_{t}(\tau)\right\|_{2}^{2}\right) \mathrm{d} \tau .
\end{gathered}
$$

By Hölder's inequality and Young's inequality again, it follows from (86), (88), and (97) that

$$
\begin{aligned}
K^{\prime}(t) \leq & K(t)+\left\|\nabla u_{0}\right\|_{2}^{2}+\left\|\nabla v_{0}\right\|_{2}^{2}+\left\|u_{t}\right\|_{2}^{2}+\left\|v_{t}\right\|_{2}^{2} \\
& +\int_{0}^{t}\left(\left\|\nabla u_{t}(\tau)\right\|_{2}^{2}+\left\|\nabla v_{t}(\tau)\right\|_{2}^{2}\right) \mathrm{d} \tau .
\end{aligned}
$$

In view of (95) and (98), we have

$$
K^{\prime \prime}(t)-2(p+3) K^{\prime}(t)+2(p+3) K(t)+L_{4} \geq 0,
$$

where

$$
L_{4}:=4(p+2) E(0)+2(p+3)\left(\left\|\nabla u_{0}\right\|_{2}^{2}+\left\|\nabla v_{0}\right\|_{2}^{2}\right) \text {. }
$$

Let

$$
B(t)=K(t)+\frac{L_{4}}{2(p+3)}, \quad t>0 .
$$

Then $B(t)$ satisfies $(80)$ for $\delta^{*}=(p+1) / 2$. By (81), we see that if

$$
\begin{aligned}
K^{\prime}(0)> & (p+3-\sqrt{(p+3)(p+1)})\left[K(0)+\frac{L_{4}}{2(p+3)}\right] \\
& +\left\|\nabla u_{0}\right\|_{2}^{2}+\left\|\nabla v_{0}\right\|_{2}^{2},
\end{aligned}
$$

that is, if

$$
\begin{aligned}
E(0)< & \frac{\sqrt{p+3}}{(p+2)(\sqrt{p+3}-\sqrt{p+1})} \int_{\Omega}\left(u_{0} u_{1}+v_{0} v_{1}\right) \mathrm{d} x \\
& -\frac{p+3}{2(p+2)}\left(\left\|u_{0}\right\|_{2}^{2}+\left\|v_{0}\right\|_{2}^{2}+\left\|\nabla u_{0}\right\|_{2}^{2}+\left\|\nabla v_{0}\right\|_{2}^{2}\right),
\end{aligned}
$$

which is satisfied by the second hypothesis to Theorem 6, then we get from Lemma 10 that $K^{\prime}(t)>\left\|\nabla u_{0}\right\|_{2}^{2}+\left\|\nabla v_{0}\right\|_{2}^{2}, t>$ 0 . Thus, the proof of Lemma 12 is completed.

In what follows, we find an estimate for the life span of $K(t)$ and prove Theorem 6.

Proof of Theorem 6. Let

$$
\begin{array}{r}
h(t)=\left[K(t)+\left(T_{1}-t\right)\left(\left\|\nabla u_{0}\right\|_{2}^{2}+\left\|\nabla v_{0}\right\|_{2}^{2}\right)\right]^{-\delta^{*}}, \\
\text { for } t \in\left[0, T_{1}\right],
\end{array}
$$

where $\delta^{*}=(p+1) / 2$ and $T_{1}>0$ is a certain constant which will be specified later. Then we have

$$
\begin{gathered}
h^{\prime}(t)=-\delta^{*} h(t)^{1+1 / \delta^{*}}\left(K^{\prime}(t)-\left\|\nabla u_{0}\right\|_{2}^{2}-\left\|\nabla v_{0}\right\|_{2}^{2}\right), \\
h^{\prime \prime}(t)=-\delta^{*} h(t)^{1+2 / \delta^{*}} V(t),
\end{gathered}
$$

where

$$
\begin{aligned}
V(t)= & K^{\prime \prime}(t)\left[K(t)+\left(T_{1}-t\right)\left(\left\|\nabla u_{0}\right\|_{2}^{2}+\left\|\nabla v_{0}\right\|_{2}^{2}\right)\right] \\
& -\left(1+\delta^{*}\right)\left(K^{\prime}(t)-\left\|\nabla u_{0}\right\|_{2}^{2}-\left\|\nabla v_{0}\right\|_{2}^{2}\right)^{2} .
\end{aligned}
$$


For simplicity, we denote

$$
\begin{aligned}
& P=\|u(t)\|_{2}^{2}+\|v(t)\|_{2}^{2}, \\
& Q=\int_{0}^{t}\left(\|\nabla u(\tau)\|_{2}^{2}+\|\nabla v(\tau)\|_{2}^{2}\right) \mathrm{d} \tau, \\
& R=\left\|u_{t}(t)\right\|_{2}^{2}+\left\|v_{t}(t)\right\|_{2}^{2}, \\
& S=\int_{0}^{t}\left(\left\|\nabla u_{t}(\tau)\right\|_{2}^{2}+\left\|\nabla v_{t}(\tau)\right\|_{2}^{2}\right) \mathrm{d} \tau .
\end{aligned}
$$

It follows from (88), (96), Hölder's inequality, and Young's inequality that

$$
K^{\prime}(t) \leq 2(\sqrt{P R}+\sqrt{Q S})+\left\|\nabla u_{0}\right\|_{2}^{2}+\left\|\nabla v_{0}\right\|_{2}^{2} .
$$

By (95), we get

$$
K^{\prime \prime}(t) \geq\left(-4-8 \delta^{*}\right) E(0)+4\left(1+\delta^{*}\right)(R+S) .
$$

Applying (107)-(110), we obtain

$$
\begin{aligned}
V(t) \geq & {\left[\left(-4-8 \delta^{*}\right) E(0)+4\left(1+\delta^{*}\right)(R+S)\right] } \\
& \times\left[K(t)+\left(T_{1}-t\right)\left(\left\|\nabla u_{0}\right\|_{2}^{2}+\left\|\nabla v_{0}\right\|_{2}^{2}\right)\right] \\
& -4\left(1+\delta^{*}\right)(\sqrt{P R}+\sqrt{Q S})^{2} .
\end{aligned}
$$

From (104) and (98) we deduce that

$$
\begin{aligned}
V(t) \geq & \left(-4-8 \delta^{*}\right) E(0) h(t)^{-1 / \delta^{*}} \\
& +4\left(1+\delta^{*}\right)(R+S)\left(T_{1}-t\right)\left(\left\|\nabla u_{0}\right\|_{2}^{2}+\left\|\nabla v_{0}\right\|_{2}^{2}\right) \\
& +4\left(1+\delta^{*}\right)\left[(R+S)(P+Q)-(\sqrt{P R}+\sqrt{Q S})^{2}\right] .
\end{aligned}
$$

By Cauchy-Schwarz inequality, the last term in the above inequality is nonnegative. Hence, we have

$$
V(t) \geq\left(-4-8 \delta^{*}\right) E(0) h(t)^{-1 / \delta^{*}}, \quad t \geq 0 .
$$

Therefore by (106) and (113), we get

$$
h^{\prime \prime}(t) \leq \delta^{*}\left(4+8 \delta^{*}\right) E(0) h(t)^{1+1 / \delta^{*}}, \quad t \geq 0 .
$$

Note that by Lemma $12, h^{\prime}(t)<0$ for $t>0$. Multiplying (114) by $h^{\prime}(t)$ and integrating from 0 to $t$, we obtain

$$
h^{\prime}(t)^{2} \geq a_{1}+b_{1} h(t)^{2+1 / \delta^{*}}, \quad \text { for } t \geq 0
$$

where

$$
\begin{gathered}
a_{1}=\delta^{* 2} h(0)^{2+2 / \delta^{*}} \\
\times\left[\left(K^{\prime}(0)-\left\|\nabla u_{0}\right\|_{2}^{2}-\left\|\nabla v_{0}\right\|_{2}^{2}\right)^{2}\right. \\
\left.-8 E(0) h(0)^{-1 / \delta^{*}}\right] \\
b_{1}=8 \delta^{* 2} E(0) .
\end{gathered}
$$

We observe that $a_{1}>0$ if

$$
\begin{aligned}
E(0) & <\frac{\left(K^{\prime}(0)-\left\|\nabla u_{0}\right\|_{2}^{2}-\left\|\nabla v_{0}\right\|_{2}^{2}\right)^{2}}{8\left[K(0)+T_{1}\left(\left\|\nabla u_{0}\right\|_{2}^{2}+\left\|\nabla v_{0}\right\|_{2}^{2}\right)\right]} \\
& =\frac{\left(\int_{\Omega} u_{0} u_{1} \mathrm{~d} x+\int_{\Omega} v_{0} v_{1} \mathrm{~d} x\right)^{2}}{2\left[\left\|u_{0}\right\|_{2}^{2}+\left\|v_{0}\right\|_{2}^{2}+T_{1}\left(\left\|\nabla u_{0}\right\|_{2}^{2}+\left\|\nabla v_{0}\right\|_{2}^{2}\right)\right]} .
\end{aligned}
$$

Then by Lemma 11, there exists a finite time $T^{*}$ such that $\lim _{t \rightarrow T^{*-}} h(t)=0$. Moreover, the upper bounds of $T^{*}$ are estimated by

$$
T^{*} \leq 2^{\left(3 \delta^{*}+1\right) / 2 \delta^{*}} \frac{\delta^{*} c}{\sqrt{a_{1}}}\left\{1-[1+\operatorname{ch}(0)]^{-1 / 2 \delta^{*}}\right\} .
$$

Therefore

$$
\begin{gathered}
\lim _{t \rightarrow T^{*-}} K(t) \\
=\lim _{t \rightarrow T^{*-}}\left(\|u(t)\|_{2}^{2}+\|v(t)\|_{2}^{2}+\int_{0}^{t}\|\nabla u(\tau)\|_{2}^{2} \mathrm{~d} \tau\right. \\
\left.+\int_{0}^{t}\|\nabla v(\tau)\|_{2}^{2} \mathrm{~d} \tau\right)=\infty
\end{gathered}
$$

This completes the proof.

Remark 13. The choice of $T_{1}$ in (104) is possible provided that $T_{1} \geq T^{*}$.

\section{General Decay of Solutions}

In this section, we prove the general decay of solutions of system (1). The method of proof is similar to that of [42, Theorem 3.5]. We first state a lemma which is similar to the one first proved by Vitillaro in [33] to study a class of a scalar wave equation.

Lemma 14 ([23, Lemma 3.2]). Suppose that (20), (A1), and (A2) hold. Let $(u, v)$ be the solution of system (1). Assume further that $E(0)<E_{1}$ and

$$
\left(l_{1}\left\|\nabla u_{0}\right\|_{2}^{2}+l_{2}\left\|\nabla v_{0}\right\|_{2}^{2}\right)^{1 / 2}<\alpha_{*} .
$$

Then

$$
\begin{aligned}
& \left(l_{1}\|\nabla u(t)\|_{2}^{2}+l_{2}\|\nabla v(t)\|_{2}^{2}+\left(g_{1} \diamond \nabla u\right)(t)+\left(g_{2} \diamond \nabla v\right)(t)\right)^{1 / 2} \\
& <\alpha_{*}
\end{aligned}
$$

for all $t \in[0, T)$. 
The auxiliary functionals $I(t), J(t)$ of problem (1) are defined as

$$
\begin{aligned}
I(t):= & I(u(t), v(t))=\left(m_{0}-\int_{0}^{t} g_{1}(s) \mathrm{d} s\right)\|\nabla u(t)\|_{2}^{2} \\
& +\left(m_{0}-\int_{0}^{t} g_{2}(s) \mathrm{d} s\right)\|\nabla v(t)\|_{2}^{2} \\
& +\left(g_{1} \diamond \nabla u\right)(t)+\left(g_{2} \diamond \nabla v\right)(t) \\
& -2(p+2) \int_{\Omega} F(u, v) \mathrm{d} x, \\
J(t):= & J(u(t), v(t)) \\
= & \frac{1}{2}\left[\left(m_{0}-\int_{0}^{t} g_{1}(s) \mathrm{d} s\right)\|\nabla u\|_{2}^{2}\right. \\
& \left.+\left(m_{0}-\int_{0}^{t} g_{2}(s) \mathrm{d} s\right)\|\nabla v\|_{2}^{2}\right] \\
& +\frac{1}{2}\left[\left(g_{1} \diamond \nabla u\right)(t)+\left(g_{2} \diamond \nabla v\right)(t)\right. \\
& \left.\quad+\frac{m_{1}}{\gamma+1}\left(\|\nabla u\|_{2}^{2(\gamma+1)}+\|\nabla v\|_{2}^{2(\gamma+1)}\right)\right] \\
& -\int_{\Omega} F(u, v) \mathrm{d} x .
\end{aligned}
$$

Lemma 15. Suppose that (20), (A1), and (A2) hold. Let $(u, v)$ be the solution of system (1). Assume further that $E(0)<E_{1}$ and

$$
\left(l_{1}\left\|\nabla u_{0}\right\|_{2}^{2}+l_{2}\left\|\nabla v_{0}\right\|_{2}^{2}\right)^{1 / 2}<\alpha_{*} .
$$

Then

$$
\begin{gathered}
l\left(\|\nabla u(t)\|_{2}^{2}+\|\nabla v(t)\|_{2}^{2}\right) \leq \frac{2(p+2)}{p+1} E(t), \\
\left(g_{1} \diamond \nabla u\right)(t)+\left(g_{2} \diamond \nabla v\right)(t) \leq \frac{2(p+2)}{p+1} E(t)
\end{gathered}
$$

for all $t \in[0, T)$.

Proof. Since $E(0)<E_{1}$ and

$$
\left(l_{1}\left\|\nabla u_{0}\right\|_{2}^{2}+l_{2}\left\|\nabla v_{0}\right\|_{2}^{2}\right)^{1 / 2}<\alpha_{*},
$$

it follows from Lemma 14 and (30) that

$$
\begin{aligned}
l_{1}\|\nabla u(t)\|_{2}^{2} & +l_{2}\|\nabla v(t)\|_{2}^{2} \\
\leq & l_{1}\|\nabla u(t)\|_{2}^{2}+l_{2}\|\nabla v(t)\|_{2}^{2} \\
& +\left(g_{1} \diamond \nabla u\right)(t)+\left(g_{2} \diamond \nabla v\right)(t) \\
< & \alpha_{*}^{2}=\eta_{1}^{-1 /(p+1)},
\end{aligned}
$$

which implies that

$$
\begin{aligned}
I(t) \geq & \left(m_{0}-\int_{0}^{\infty} g_{1}(s) \mathrm{d} s\right)\|\nabla u\|_{2}^{2} \\
& +\left(m_{0}-\int_{0}^{\infty} g_{2}(s) \mathrm{d} s\right)\|\nabla v\|_{2}^{2} \\
& +\left(g_{1} \diamond \nabla u\right)(t)+\left(g_{2} \diamond \nabla v\right)(t) \\
& -2(p+2) \int_{\Omega} F(u, v) \mathrm{d} x \\
\geq & l_{1}\|\nabla u(t)\|_{2}^{2}+l_{2}\|\nabla v(t)\|_{2}^{2} \\
& -2(p+2) \int_{\Omega} F(u, v) \mathrm{d} x \\
= & l_{1}\|\nabla u(t)\|_{2}^{2}+l_{2}\|\nabla v(t)\|_{2}^{2} \\
& -\left(a\|u+v\|_{2(p+2)}^{2(p+2)}+2 b\|u v\|_{p+2}^{p+2}\right) \\
\geq & l_{1}\|\nabla u(t)\|_{2}^{2}+l_{2}\|\nabla v(t)\|_{2}^{2} \\
& -\eta_{1}\left(l_{1}\|\nabla u(t)\|_{2}^{2}+l_{2}\|\nabla v(t)\|_{2}^{2}\right)^{p+2} \\
= & \left(l_{1}\|\nabla u(t)\|_{2}^{2}+l_{2}\|\nabla v(t)\|_{2}^{2}\right) \\
& \times\left[1-\eta_{1}\left(l_{1}\|\nabla u(t)\|_{2}^{2}+l_{2}\|\nabla v(t)\|_{2}^{2}\right)^{p+1}\right] \geq 0
\end{aligned}
$$

for $t \in[0, T)$, where we have used (24). Further, by (123), we have

$$
\begin{aligned}
J(t) \geq \frac{1}{2}[ & \left(m_{0}-\int_{0}^{t} g_{1}(s) \mathrm{d} s\right)\|\nabla u\|_{2}^{2} \\
& +\left(m_{0}-\int_{0}^{t} g_{2}(s) \mathrm{d} s\right)\|\nabla v\|_{2}^{2}+\left(g_{1} \diamond \nabla u\right)(t) \\
& \left.\quad+\left(g_{2} \diamond \nabla v\right)(t)\right] \\
& -\int_{\Omega} F(u, v) \mathrm{d} x-\frac{1}{2(p+2)} I(t)+\frac{1}{2(p+2)} I(t) \\
\geq & \left(\frac{1}{2}-\frac{1}{2(p+2)}\right) \\
\times & {\left[\left(m_{0}-\int_{0}^{t} g_{1}(s) \mathrm{d} s\right)\|\nabla u\|_{2}^{2}\right.} \\
& \left.+\left(m_{0}-\int_{0}^{t} g_{2}(s) \mathrm{d} s\right)\|\nabla v\|_{2}^{2}\right] \\
+ & \left(\frac{1}{2}-\frac{1}{2(p+2)}\right)\left[\left(g_{1} \diamond \nabla u\right)(t)+\left(g_{2} \diamond \nabla v\right)(t)\right] \\
+ & \frac{1}{2(p+2)} I(t)
\end{aligned}
$$




$$
\begin{aligned}
& \geq \frac{p+1}{2(p+2)} \\
& \quad \times\left[l_{1}\|\nabla u(t)\|_{2}^{2}+l_{2}\|\nabla v(t)\|_{2}^{2}\right. \\
& \left.\quad+\left(g_{1} \diamond \nabla u\right)(t)+\left(g_{2} \diamond \nabla v\right)(t)\right] \\
& \quad+\frac{1}{2(p+2)} I(t) \\
& \geq 0 .
\end{aligned}
$$

From (130) and (36), we deduce that

$$
\begin{aligned}
& l\left(\|\nabla u(t)\|_{2}^{2}+\|\nabla v(t)\|_{2}^{2}\right) \\
& \quad \leq l_{1}\|\nabla u(t)\|_{2}^{2}+l_{2}\|\nabla v(t)\|_{2}^{2} \leq \frac{2(p+2)}{p+1} J(t) \\
& \quad \leq \frac{2(p+2)}{p+1} E(t) \\
& \left(g_{1} \diamond \nabla u\right)(t) \\
& \quad+\left(g_{2} \diamond \nabla v\right)(t) \leq \frac{2(p+2)}{p+1} J(t) \leq \frac{2(p+2)}{p+1} E(t) .
\end{aligned}
$$
[42]:

We note that the following functional was introduced in

$$
G_{1}(t):=E(t)+\varepsilon_{1} \phi(t)+\varepsilon_{2} \psi(t)
$$

where $\varepsilon_{1}$ and $\varepsilon_{2}$ are some positive constants and

$$
\begin{aligned}
\phi(t)= & \xi_{1}(t) \int_{\Omega} u(t) u_{t}(t) \mathrm{d} x+\xi_{2}(t) \int_{\Omega} v(t) v_{t}(t) \mathrm{d} x, \\
\psi(t)= & -\xi_{1}(t) \int_{\Omega} u_{t}(t) \int_{0}^{t} g_{1}(t-s)(u(t)-u(s)) \mathrm{d} s \mathrm{~d} x \\
& -\xi_{2}(t) \int_{\Omega} v_{t}(t) \int_{0}^{t} g_{2}(t-s)(v(t)-v(s)) \mathrm{d} s \mathrm{~d} x .
\end{aligned}
$$

Here, we use the same functional (132) but choose $\xi_{i}(t) \equiv$ $1(i=1,2)$ in (133) and (134).

Lemma 16. There exist two positive constants $\beta_{1}$ and $\beta_{2}$ such that

$$
\beta_{1} G_{1}(t) \leq E(t) \leq \beta_{2} G_{1}(t), \quad \forall t \geq 0 .
$$

Proof. From Hölder's inequality, Young's inequality, Lemma 2, (36), and (125), we deduce

$$
\begin{aligned}
|\phi(t)| & \leq\|u\|_{2}\left\|u_{t}\right\|_{2}+\|v\|_{2}\left\|v_{t}\right\|_{2} \\
& \leq \frac{1}{2}\left(\|u\|_{2}^{2}+\left\|u_{t}\right\|_{2}^{2}+\|v\|_{2}^{2}+\left\|v_{t}\right\|_{2}^{2}\right) \\
& \leq \frac{1}{2} C^{2}\left(\|\nabla u\|_{2}^{2}+\|\nabla v\|_{2}^{2}\right)+\frac{1}{2}\left(\left\|u_{t}\right\|_{2}^{2}+\left\|v_{t}\right\|_{2}^{2}\right) \\
& \leq C^{2} \frac{p+2}{l(p+1)} E(t)+E(t)=\left(1+C^{2} \frac{p+2}{l(p+1)}\right) E(t) .
\end{aligned}
$$

Applying Hölder's inequality, Young's inequality, and Lemma 2 again, it follows that

$$
\begin{aligned}
\int_{\Omega} u_{t}( & t) \int_{0}^{t} g_{1}(t-s)(u(t)-u(s)) \mathrm{d} s \mathrm{~d} x \\
\leq & \left\|u_{t}\right\|_{2} \\
& \times\left[\int_{\Omega}\left(\int_{0}^{t} g_{1}(t-s)(u(t)-u(s)) \mathrm{d} s\right)^{2} \mathrm{~d} x\right]^{1 / 2} \\
\leq & \frac{1}{2}\left\|u_{t}\right\|_{2}^{2}+\frac{1}{2} \int_{\Omega}\left(\int_{0}^{t} g_{1}(t-s)(u(t)-u(s)) \mathrm{d} s\right)^{2} \mathrm{~d} x \\
\leq & \frac{1}{2}\left\|u_{t}\right\|_{2}^{2} \\
& +\frac{1}{2}\left(m_{0}-l\right) \int_{\Omega} \int_{0}^{t} g_{1}(t-s)(u(t)-u(s))^{2} \mathrm{~d} s \mathrm{~d} x \\
\leq & \frac{1}{2}\left\|u_{t}\right\|_{2}^{2}+\frac{1}{2} C^{2}\left(m_{0}-l\right)\left(g_{1} \diamond \nabla u\right)(t) .
\end{aligned}
$$

Similarly, applying Hölder's inequality, Young's inequality, and Lemma 2 again, we have

$$
\begin{aligned}
\int_{\Omega} v_{t}(t) \int_{0}^{t} g_{2}(t-s)(v(t)-v(s)) \mathrm{d} s \mathrm{~d} x \\
\quad \leq \frac{1}{2}\left\|v_{t}(t)\right\|_{2}^{2}+\frac{1}{2} C^{2}\left(m_{0}-l\right)\left(g_{2} \diamond \nabla v\right)(t) .
\end{aligned}
$$

It follows from (137), (138), (36), and (126) that

$$
\begin{aligned}
|\psi(t)| \leq & \frac{1}{2}\left(\left\|u_{t}\right\|_{2}^{2}+\left\|v_{t}\right\|_{2}^{2}\right) \\
& +\frac{1}{2} C^{2}\left(m_{0}-l\right)\left[\left(g_{1} \diamond \nabla u\right)(t)+\left(g_{2} \diamond \nabla v\right)(t)\right] \\
\leq & E(t)+\frac{p+2}{p+1} C^{2}\left(m_{0}-l\right) E(t) \\
= & \left(1+\frac{p+2}{p+1} C^{2}\left(m_{0}-l\right)\right) E(t) .
\end{aligned}
$$

If we take $\epsilon_{1}$ and $\epsilon_{2}$ to be sufficiently small, then (135) follows from (132), (136), and (139). 
Lemma 17. Under the conditions of Theorem 7, the functional $\phi(t)$, defined by $(133)$ (with $\xi_{i}(t) \equiv 1(i=1,2)$ ), satisfies

$$
\begin{aligned}
\phi^{\prime}(t) \leq & \left\|u_{t}\right\|_{2}^{2}+\left\|v_{t}\right\|_{2}^{2}-\left[l-\epsilon\left(m_{0}-l+\frac{1}{2}\right)\right]\|\nabla u\|_{2}^{2} \\
& -\left[l-\epsilon\left(m_{0}-l+\frac{1}{2}\right)\right]\|\nabla v\|_{2}^{2} \\
& +\frac{1}{4 \epsilon}\left[\left(g_{1} \diamond \nabla u\right)(t)+\left(g_{2} \diamond \nabla v\right)(t)\right] \\
& +\frac{1}{2 \epsilon}\left(\left\|\nabla u_{t}\right\|_{2}^{2}+\left\|\nabla v_{t}\right\|_{2}^{2}\right) \\
& +2(p+2) \int_{\Omega} F(u, v) \mathrm{d} x \\
& -m_{1}\left(\|\nabla u\|_{2}^{2(\gamma+1)}+\|\nabla v\|_{2}^{2(\gamma+1)}\right), \quad \forall \epsilon>0 .
\end{aligned}
$$

Proof. By using the equations in (1) and setting $\xi_{i}(t) \equiv 1(i=$ $1,2)$ in (133), we easily see that

$$
\begin{aligned}
\phi^{\prime}(t)= & \left\|u_{t}\right\|_{2}^{2}+\left\|v_{t}\right\|_{2}^{2}+\int_{\Omega} u u_{t t} \mathrm{~d} x+\int_{\Omega} v v_{t t} \mathrm{~d} x \\
= & \left\|u_{t}\right\|_{2}^{2}+\left\|v_{t}\right\|_{2}^{2} \\
& -M\left(\|\nabla u\|_{2}^{2}\right)\|\nabla u\|_{2}^{2}-M\left(\|\nabla v\|_{2}^{2}\right)\|\nabla v\|_{2}^{2} \\
& +\int_{\Omega} \nabla u(t) \cdot \int_{0}^{t} g_{1}(t-s) \nabla u(s) \mathrm{d} s \mathrm{~d} x \\
& +\int_{\Omega} \nabla v(t) \cdot \int_{0}^{t} g_{2}(t-s) \nabla v(s) \mathrm{d} s \mathrm{~d} x \\
& -\int_{\Omega} \nabla u_{t} \cdot \nabla u \mathrm{~d} x-\int_{\Omega} \nabla v_{t} \cdot \nabla v \mathrm{~d} x+2(p+2) \\
& \times \int_{\Omega} F(u, v) \mathrm{d} x .
\end{aligned}
$$

By Cauchy-Schwarz inequality and Young's inequality, we have

$$
\begin{aligned}
\int_{\Omega} \nabla & u(t) \cdot \int_{0}^{t} g_{1}(t-s) \nabla u(s) \mathrm{d} s \mathrm{~d} x \\
\leq & \left(\int_{0}^{t} g_{1}(t-s) \mathrm{d} s\right)\|\nabla u(t)\|_{2}^{2} \\
& +\int_{\Omega} \int_{0}^{t} g_{1}(t-s)|\nabla u(t)| \\
& \times|\nabla u(s)-\nabla u(t)| \mathrm{d} s \mathrm{~d} x \leq(1+\epsilon)\left(\int_{0}^{t} g_{1}(s) \mathrm{d} s\right) \\
& \times\|\nabla u(t)\|_{2}^{2}+\frac{1}{4 \epsilon}\left(g_{1} \diamond \nabla u\right)(t) \leq(1+\epsilon)\left(m_{0}-l\right) \\
& \times\|\nabla u(t)\|_{2}^{2}+\frac{1}{4 \epsilon}\left(g_{1} \diamond \nabla u\right)(t), \quad \forall \epsilon>0 .
\end{aligned}
$$

In the same way, we can get

$$
\begin{aligned}
& \int_{\Omega} \nabla v(t) \cdot \int_{0}^{t} g_{2}(t-s) \nabla v(s) \mathrm{d} s \mathrm{~d} x \\
& \leq(1+\epsilon)\left(m_{0}-l\right)\|\nabla v(t)\|_{2}^{2}+\frac{1}{4 \epsilon}\left(g_{2} \diamond \nabla v\right)(t), \\
& \quad \int_{\Omega} \nabla u_{t} \cdot \nabla u \mathrm{~d} x \leq \frac{\epsilon}{2}\|\nabla u\|_{2}^{2}+\frac{1}{2 \epsilon}\left\|\nabla u_{t}\right\|_{2}^{2}, \\
& \int_{\Omega} \nabla v_{t} \cdot \nabla v \mathrm{~d} x \leq \frac{\epsilon}{2}\|\nabla v\|_{2}^{2}+\frac{1}{2 \epsilon}\left\|\nabla v_{t}\right\|_{2}^{2}, \quad \forall \epsilon>0 .
\end{aligned}
$$

Using (141)-(145), we obtain

$$
\begin{aligned}
\phi^{\prime}(t) \leq & \left\|u_{t}\right\|_{2}^{2}+\left\|v_{t}\right\|_{2}^{2}-m_{0}\|\nabla u\|_{2}^{2} \\
& -m_{0}\|\nabla v\|_{2}^{2}-m_{1}\left(\|\nabla u\|_{2}^{2(\gamma+1)}+\|\nabla v\|_{2}^{2(\gamma+1)}\right) \\
& +(1+\epsilon)\left(m_{0}-l\right)\left(\|\nabla u\|_{2}^{2}+\|\nabla v(t)\|_{2}^{2}\right) \\
& +\frac{1}{4 \epsilon}\left(\left(g_{1} \diamond \nabla u\right)(t)+\left(g_{2} \diamond \nabla v\right)(t)\right) \\
& +\frac{\epsilon}{2}\left(\|\nabla u\|_{2}^{2}+\|\nabla v\|_{2}^{2}\right)+\frac{1}{2 \epsilon}\left(\left\|\nabla u_{t}\right\|_{2}^{2}+\left\|\nabla v_{t}\right\|_{2}^{2}\right) \\
& +2(p+2) \int_{\Omega} F(u, v) \mathrm{d} x \\
= & \left\|u_{t}\right\|_{2}^{2}+\left\|v_{t}\right\|_{2}^{2} \\
& -\left[l-\epsilon\left(m_{0}-l+\frac{1}{2}\right)\right]\|\nabla u\|_{2}^{2}-\left[l-\epsilon\left(m_{0}-l+\frac{1}{2}\right)\right] \\
& \times\|\nabla v\|_{2}^{2}+\frac{1}{4 \epsilon}\left(g_{1} \diamond \nabla u\right)(t)+\frac{1}{4 \epsilon}\left(g_{2} \diamond \nabla v\right)(t) \\
& +\frac{1}{2 \epsilon}\left(\left\|\nabla u_{t}\right\|_{2}^{2}+\left\|\nabla v_{t}\right\|_{2}^{2}\right) \\
& -m_{1}\left(\|\nabla u\|_{2}^{2(\gamma+1)}+\|\nabla v\|_{2}^{2(\gamma+1)}\right) \\
& 2(p+2) \int_{\Omega} F(u, v) \mathrm{d} x, \forall \epsilon>0 .
\end{aligned}
$$

So, Lemma 17 is established.

Lemma 18. Under the conditions of Theorem 7 , the functional $\psi(t)$, defined by $(134)$ (with $\left.\xi_{i}(t) \equiv 1(i=1,2)\right)$, satisfies

$$
\begin{aligned}
\psi^{\prime}(t) \leq & {\left[\eta\left(m_{0}-l\right)\left(3 m_{0}-2 l\right)+3 C \eta C_{7}\right] } \\
& \times\left(\|\nabla u\|_{2}^{2}+\|\nabla v\|_{2}^{2}\right)+\eta\left(\left\|\nabla u_{t}\right\|_{2}^{2}+\left\|\nabla v_{t}\right\|_{2}^{2}\right) \\
& +\eta m_{1}\left(m_{0}-l\right)\left(\|\nabla u\|_{2}^{2(\gamma+1)}+\|\nabla v\|_{2}^{2(\gamma+1)}\right) \\
& +\left[\left(2 \eta+\frac{2+C^{2}}{4 \eta}\right)\left(m_{0}-l\right)+\frac{C_{6}}{4 \eta}\right] \\
& \times\left[\left(g_{1} \diamond \nabla u\right)(t)+\left(g_{2} \diamond \nabla v\right)(t)\right]
\end{aligned}
$$


Journal of Function Spaces

17

$$
\begin{aligned}
& -\frac{g_{0}(0)}{4 \eta} C^{2}\left[\left(g_{1}^{\prime} \diamond \nabla u\right)(t)+\left(g_{2}^{\prime} \diamond \nabla v\right)(t)\right] \\
& +\left(\eta-\int_{0}^{t} g_{1}(s) d s\right)\left\|u_{t}\right\|_{2}^{2} \\
& +\left(\eta-\int_{0}^{t} g_{2}(s) d s\right)\left\|v_{t}\right\|_{2}^{2}, \quad \forall \eta>0,
\end{aligned}
$$

where

$$
\begin{aligned}
& g_{0}(0)=\max \left\{g_{1}(0), g_{2}(0)\right\}, \\
& C_{6}:=m_{0}+m_{1}\left(\frac{2(p+2)}{l(p+1)} E(0)\right)^{\gamma}, \\
& C_{7}:=C^{2(2 p+3)}\left[\frac{2(p+2)}{l(p+1)} E(0)\right]^{2(p+1)} .
\end{aligned}
$$

Proof. By using the equations in (1) and set $\xi_{i}(t) \equiv 1(i=$ $1,2)$ in (134), we observe that

$$
\begin{aligned}
\psi^{\prime}(t)= & -\int_{\Omega} u_{t t}(t) \int_{0}^{t} g_{1}(t-s)(u(t)-u(s)) \mathrm{d} s \mathrm{~d} x \\
& -\int_{\Omega} v_{t t}(t) \int_{0}^{t} g_{2}(t-s)(v(t)-v(s)) \mathrm{d} s \mathrm{~d} x \\
& -\int_{\Omega} u_{t}(t) \int_{0}^{t} g_{1}^{\prime}(t-s)(u(t)-u(s)) \mathrm{d} s \mathrm{~d} x \\
& -\int_{\Omega} v_{t}(t) \int_{0}^{t} g_{2}^{\prime}(t-s)(v(t)-v(s)) \mathrm{d} s \mathrm{~d} x \\
& -\int_{\Omega} u_{t}(t) \int_{0}^{t} g_{1}(t-s) u_{t}(t) \mathrm{d} s \mathrm{~d} x \\
& -\int_{\Omega} v_{t}(t) \int_{0}^{t} g_{2}(t-s) v_{t}(t) \mathrm{d} s \mathrm{~d} x \\
= & \int_{\Omega} M\left(\|\nabla u\|_{2}^{2}\right) \nabla u(t) \\
& \cdot \int_{0}^{t} g_{1}(t-s)(\nabla u(t)-\nabla u(s)) \mathrm{d} s \mathrm{~d} x \\
& +\int_{\Omega}^{t}\left(\int_{0}^{t} g_{1}(t-s)(\nabla u(t)-\nabla u(s)) \mathrm{d} s\right] \mathrm{d} x \\
& \cdot \int_{0}^{t} g_{2}(t-s)(\nabla v(t)-\nabla v(s)) \mathrm{d} s \mathrm{~d} x \\
& \\
& \\
& \\
& \\
&
\end{aligned}
$$

$$
\begin{aligned}
& -\int_{\Omega}\left(\int_{0}^{t} g_{2}(t-s) \nabla v(s) \mathrm{d} s\right) \\
& \times\left[\int_{0}^{t} g_{2}(t-s)(\nabla v(t)-\nabla v(s)) \mathrm{d} s\right] \mathrm{d} x \\
& +\left[\int_{\Omega} \nabla u_{t}(t) \int_{0}^{t} g_{1}(t-s)(\nabla u(t)-\nabla u(s)) \mathrm{d} s \mathrm{~d} x\right. \\
& \left.+\int_{\Omega} \nabla v_{t}(t) \int_{0}^{t} g_{2}(t-s)(\nabla v(t)-\nabla v(s)) \mathrm{d} s \mathrm{~d} x\right] \\
& -\left[\int_{\Omega} f_{1}(u, v) \int_{0}^{t} g_{1}(t-s)(u(t)-u(s)) \mathrm{d} s \mathrm{~d} x\right. \\
& \left.+\int_{\Omega} f_{2}(u, v) \int_{0}^{t} g_{2}(t-s)(v(t)-v(s)) \mathrm{d} s \mathrm{~d} x\right] \\
& -\left[\int_{\Omega} u_{t}(t) \int_{0}^{t} g_{1}^{\prime}(t-s)(u(t)-u(s)) \mathrm{d} s \mathrm{~d} x\right. \\
& \left.+\int_{\Omega} v_{t}(t) \int_{0}^{t} g_{2}^{\prime}(t-s)(v(t)-v(s)) \mathrm{d} s \mathrm{~d} x\right] \\
& -\left(\int_{0}^{t} g_{1}(s) \mathrm{d} s\right)\left\|u_{t}(t)\right\|_{2}^{2} \\
& -\left(\int_{0}^{t} g_{2}(s) \mathrm{d} s\right)\left\|v_{t}(t)\right\|_{2}^{2} \\
& :=I_{1}+\cdots+I_{7}-\left(\int_{0}^{t} g_{1}(s) \mathrm{d} s\right)\left\|u_{t}(t)\right\|_{2}^{2} \\
& -\left(\int_{0}^{t} g_{2}(s) \mathrm{d} s\right)\left\|v_{t}(t)\right\|_{2}^{2} .
\end{aligned}
$$

In what follows we will estimate $I_{1}, \ldots, I_{7}$ in (149). By Young's inequality, (A2), and (125), we get

$$
\begin{aligned}
\left|I_{1}\right| \leq & \eta M\left(\|\nabla u\|_{2}^{2}\right)\|\nabla u\|_{2}^{2} \int_{0}^{t} g_{1}(s) \mathrm{d} s \\
& +\frac{1}{4 \eta} M\left(\|\nabla u\|_{2}^{2}\right)\left(g_{1} \diamond \nabla u\right)(t) \\
= & \eta\left(m_{0}\|\nabla u\|_{2}^{2}+m_{1}\|\nabla u\|_{2}^{2(\gamma+1)}\right) \int_{0}^{t} g_{1}(s) \mathrm{d} s \\
& +\frac{1}{4 \eta}\left(m_{0}+m_{1}\|\nabla u\|_{2}^{2 \gamma}\right)\left(g_{1} \diamond \nabla u\right)(t) \\
\leq & \eta\left(m_{0}-l\right)\left(m_{0}\|\nabla u\|_{2}^{2}+m_{1}\|\nabla u\|_{2}^{2(\gamma+1)}\right) \\
& +\frac{1}{4 \eta}\left[m_{0}+m_{1}\left(\frac{2(p+2)}{l(p+1)} E(0)\right)^{\gamma}\right]\left(g_{1} \diamond \nabla u\right)(t) \\
\leq & \eta m_{0}\left(m_{0}-l\right)\|\nabla u\|_{2}^{2}+\eta m_{1}\left(m_{0}-l\right)\|\nabla u\|_{2}^{2(\gamma+1)} \\
& +\frac{C_{6}}{4 \eta}\left(g_{1} \diamond \nabla u\right)(t), \quad \forall \eta>0 .
\end{aligned}
$$


Similarly, we have

$$
\begin{gathered}
\left|I_{2}\right| \leq \eta m_{0}\left(m_{0}-l\right)\|\nabla v\|_{2}^{2}+\eta m_{1}\left(m_{0}-l\right)\|\nabla v\|_{2}^{2(\gamma+1)} \\
+\frac{C_{6}}{4 \eta}\left(g_{2} \diamond \nabla v\right)(t), \quad \forall \eta>0 .
\end{gathered}
$$

For $I_{3}$ in (149), applying (A2), Hölder's inequality, and Young's inequality, we deduce

$$
\begin{aligned}
\left|I_{3}\right| \leq & \eta \int_{\Omega}\left(\int_{0}^{t} g_{1}(t-s) \nabla u(s) \mathrm{d} s\right)^{2} \mathrm{~d} x \\
& +\frac{1}{4 \eta} \int_{\Omega}\left(\int_{0}^{t} g_{1}(t-s)(\nabla u(t)-\nabla u(s)) \mathrm{d} s\right)^{2} \mathrm{~d} x \\
\leq & \eta \int_{\Omega}\left[\int_{0}^{t} g_{1}(t-s)(|\nabla u(s)-\nabla u(t)|+|\nabla u(t)|) \mathrm{d} s\right]^{2} \mathrm{~d} x \\
& +\frac{1}{4 \eta}\left(m_{0}-l\right)\left(g_{1} \diamond \nabla u\right)(t) \\
\leq & 2 \eta\left(\int_{0}^{t} g_{1}(s) \mathrm{d} s\right)^{2}\|\nabla u\|_{2}^{2} \\
& +\left(2 \eta+\frac{1}{4 \eta}\right)\left(m_{0}-l\right)\left(g_{1} \diamond \nabla u\right)(t) \\
\leq & 2 \eta\left(m_{0}-l\right)^{2}\|\nabla u\|_{2}^{2} \\
& +\left(2 \eta+\frac{1}{4 \eta}\right)\left(m_{0}-l\right)\left(g_{1} \diamond \nabla u\right)(t), \quad \forall \eta>0 .
\end{aligned}
$$

Similarly,

$$
\begin{aligned}
\left|I_{4}\right| \leq & 2 \eta\left(m_{0}-l\right)^{2}\|\nabla v\|_{2}^{2} \\
& +\left(2 \eta+\frac{1}{4 \eta}\right)\left(m_{0}-l\right)\left(g_{2} \diamond \nabla v\right)(t), \quad \forall \eta>0 .
\end{aligned}
$$

In the same way, we have

$$
\begin{aligned}
\left|I_{5}\right| \leq & \eta\left(\left\|\nabla u_{t}\right\|_{2}^{2}+\left\|\nabla v_{t}\right\|_{2}^{2}\right) \\
& +\frac{1}{4 \eta}\left(m_{0}-l\right)\left[\left(g_{1} \diamond \nabla u\right)(t)+\left(g_{2} \diamond \nabla v\right)(t)\right],
\end{aligned}
$$

$\forall \eta>0$.

By Young's inequality, Lemma 2, and (125) we see that

$$
\begin{gathered}
\left|\int_{\Omega} f_{1}(u, v) \int_{0}^{t} g_{1}(t-s)(u(t)-u(s)) \mathrm{d} s \mathrm{dx}\right| \\
\leq \eta \int_{\Omega}\left(a|u+v|^{2 p+3}+b|u|^{p+1}|v|^{p+2}\right)^{2} \mathrm{~d} x
\end{gathered}
$$

$$
\begin{aligned}
& +\frac{C^{2}\left(m_{0}-l\right)}{4 \eta}\left(g_{1} \diamond \nabla u\right)(t) \\
& \leq C \eta \int_{\Omega}\left(|u|^{2(2 p+3)}+|v|^{2(2 p+3)}+|u|^{2(p+1)}|v|^{2(p+2)}\right) \mathrm{d} x \\
& +\frac{C^{2}\left(m_{0}-l\right)}{4 \eta}\left(g_{1} \diamond \nabla u\right)(t) \\
& \leq C \eta\left[\|u\|_{2(2 p+3)}^{2(2 p+3)}+\|v\|_{2(2 p+3)}^{2(2 p+3)}+\|u\|_{2(2 p+3)}^{2(p+1)}\|v\|_{2(2 p+3)}^{2(p+2)}\right] \\
& +\frac{C^{2}\left(m_{0}-l\right)}{4 \eta}\left(g_{1} \diamond \nabla u\right)(t) \\
& \leq C \eta C^{2(2 p+3)} \\
& \times\left[\|\nabla u\|_{2}^{2(2 p+3)}+\|\nabla v\|_{2}^{2(2 p+3)}+\|\nabla u\|_{2}^{2(p+1)}\|\nabla v\|_{2}^{2(p+2)}\right] \\
& +\frac{C^{2}\left(m_{0}-l\right)}{4 \eta}\left(g_{1} \diamond \nabla u\right)(t) \\
& \leq C \eta C^{2(2 p+3)}\left(\frac{2(p+2)}{l(p+1)} E(0)\right)^{2 p+2} \\
& \times\left[\|\nabla u\|_{2}^{2}+\|\nabla v\|_{2}^{2}+\|\nabla u\|_{2}\|\nabla v\|_{2}\right] \\
& +\frac{C^{2}\left(m_{0}-l\right)}{4 \eta}\left(g_{1} \diamond \nabla u\right)(t) \\
& \leq \frac{3}{2} C \eta C_{7}\left[\|\nabla u\|_{2}^{2}+\|\nabla v\|_{2}^{2}\right] \\
& +\frac{C^{2}\left(m_{0}-l\right)}{4 \eta}\left(g_{1} \diamond \nabla u\right)(t), \quad \forall \eta>0 .
\end{aligned}
$$

Hence, we infer that

$$
\begin{aligned}
\left|I_{6}\right| \leq & 3 C \eta C_{7}\left(\|\nabla u\|_{2}^{2}+\|\nabla v\|_{2}^{2}\right) \\
& +\frac{C^{2}\left(m_{0}-l\right)}{4 \eta}\left[\left(g_{1} \diamond \nabla u\right)(t)+\left(g_{2} \diamond \nabla v\right)(t)\right],
\end{aligned}
$$

$$
\forall \eta>0
$$

By Young's inequality and Lemma 2 again, we obtain

$$
\begin{aligned}
\left|I_{7}\right| \leq & \eta\left\|u_{t}\right\|_{2}^{2}+\frac{1}{4 \eta} \int_{\Omega}\left(\int_{0}^{t} g_{1}^{\prime}(t-s)(u(t)-u(s)) \mathrm{d} s\right)^{2} \mathrm{~d} x \\
& +\eta\left\|v_{t}\right\|_{2}^{2}+\frac{1}{4 \eta} \int_{\Omega}\left(\int_{0}^{t} g_{2}^{\prime}(t-s)(v(t)-v(s)) \mathrm{d} s\right)^{2} \mathrm{~d} x \\
\leq & \eta\left(\left\|u_{t}\right\|_{2}^{2}+\left\|v_{t}\right\|_{2}^{2}\right)+\frac{C^{2}}{4 \eta}\left(\int_{0}^{t} g_{1}^{\prime}(s) \mathrm{d} s\right)\left(g_{1}^{\prime} \diamond \nabla u\right)(t) \\
& +\frac{C^{2}}{4 \eta}\left(\int_{0}^{t} g_{2}^{\prime}(s) \mathrm{d} s\right)\left(g_{2}^{\prime} \diamond \nabla v\right)(t)
\end{aligned}
$$




$$
\begin{gathered}
\leq \eta\left(\left\|u_{t}\right\|_{2}^{2}+\left\|v_{t}\right\|_{2}^{2}\right)-\frac{g_{1}(0) C^{2}}{4 \eta}\left(g_{1}^{\prime} \diamond \nabla u\right)(t) \\
-\frac{g_{2}(0) C^{2}}{4 \eta}\left(g_{2}^{\prime} \diamond \nabla v\right)(t), \quad \forall \eta>0 .
\end{gathered}
$$

Combining (149)-(157), we complete the proof of Lemma 18.

Proof of Theorem 7. Since $g_{i}$ are positive, we have, for any $t_{0}>$ 0 ,

$$
\int_{0}^{t} g_{i}(s) \mathrm{d} s \geq \int_{0}^{t_{0}} g_{i}(s) \mathrm{d} s \quad(i=1,2), t \geq t_{0},
$$

denote

$$
g_{3}=\min \left\{\int_{0}^{t_{0}} g_{1}(s) \mathrm{d} s, \int_{0}^{t_{0}} g_{2}(s) \mathrm{d} s\right\} .
$$

By using (28), (132), (140), and (147), a series of computations, for $t \geq t_{0}$, we have

$$
\begin{aligned}
G_{1}^{\prime}(t)= & E^{\prime}(t)+\varepsilon_{1} \phi^{\prime}(t)+\varepsilon_{2} \psi^{\prime}(t) \\
\leq & -\left\{\varepsilon_{1}\left[l-\epsilon\left(m_{0}-l+\frac{1}{2}\right)\right]\right. \\
& \left.-\varepsilon_{2} \eta\left[\left(m_{0}-l\right)\left(3 m_{0}-2 l\right)+3 C C_{7}\right]\right\} \\
& \times\left[\|\nabla u\|_{2}^{2}+\|\nabla v\|_{2}^{2}\right] \\
& -\left[m_{1} \varepsilon_{1}-m_{1} \varepsilon_{2} \eta\left(m_{0}-l\right)\right] \\
& \times\left(\|\nabla u\|_{2}^{2(\gamma+1)}+\|\nabla v\|_{2}^{2(\gamma+1)}\right) \\
& -\left(1-\frac{\varepsilon_{1}}{2 \epsilon}-\varepsilon_{2} \eta\right)\left(\left\|\nabla u_{t}\right\|_{2}^{2}+\left\|\nabla v_{t}\right\|_{2}^{2}\right) \\
& +\left\{\frac{\varepsilon_{1}}{4 \epsilon}+\varepsilon_{2}\left[\left(2 \eta+\frac{2+C^{2}}{4 \eta}\right)\left(m_{0}-l\right)+\frac{C_{6}}{4 \eta}\right]\right\} \\
& \times\left[\left(g_{1} \diamond \nabla u\right)(t)+\left(g_{2} \diamond \nabla v\right)(t)\right] \\
& -\left(\varepsilon_{2} g_{3}-\varepsilon_{2} \eta-\varepsilon_{1}\right)\left(\left\|u_{t}\right\|_{2}^{2}+\left\|v_{t}\right\|_{2}^{2}\right) \\
& +2 \varepsilon_{1}(p+2) \int_{\Omega} F(u, v) \mathrm{d} x \\
& +\left(\frac{1}{2}-\frac{g_{0}(0)}{4 \eta} \varepsilon_{2} C^{2}\right) \\
& \times\left[\left(g_{1}^{\prime} \diamond \nabla u\right)(t)+\left(g_{2}^{\prime} \diamond \nabla v\right)(t)\right] .
\end{aligned}
$$

We choose $\varepsilon_{1}, \varepsilon_{2}$, and $\eta$ small enough, such that

$$
\varepsilon_{2} \eta\left(m_{0}-l\right)<\varepsilon_{1}<\varepsilon_{2}\left(g_{3}-\eta\right)
$$

and $\epsilon=\varepsilon_{1}$; then we can check that

$$
\begin{aligned}
& C_{8}=\varepsilon_{2} g_{3}-\varepsilon_{2} \eta-\varepsilon_{1}>0, \\
& C_{10}=m_{1} \varepsilon_{1}-m_{1} \varepsilon_{2} \eta\left(m_{0}-l\right)>0, \\
& C_{11}=\frac{1}{2}-\frac{g_{0}(0)}{4 \eta} \varepsilon_{2} C^{2}>0, \\
& C_{12}=1-\frac{\varepsilon_{1}}{2 \epsilon}-\varepsilon_{2} \eta>0 .
\end{aligned}
$$

We choose $\eta$, $\varepsilon_{1}$, and $\varepsilon_{2}$ so small that (162) remains valid and, further

$$
\begin{aligned}
C_{9}= & \varepsilon_{1}\left[l-\epsilon\left(m_{0}-l+\frac{1}{2}\right)\right] \\
& -\varepsilon_{2} \eta\left[\left(m_{0}-l\right)\left(3 m_{0}-2 l\right)+3 C C_{7}\right]>0 .
\end{aligned}
$$

So, we arrive at

$$
\begin{aligned}
G_{1}^{\prime}(t) \leq & -C_{8}\left(\left\|u_{t}\right\|_{2}^{2}+\left\|v_{t}\right\|_{2}^{2}\right) \\
& -C_{9}\left(\|\nabla u\|_{2}^{2}+\|\nabla v\|_{2}^{2}\right)+2 \varepsilon_{1}(p+2) \int_{\Omega} F(u, v) \mathrm{d} x \\
& -C_{10}\left(\|\nabla u\|_{2}^{2(\gamma+1)}+\|\nabla v\|_{2}^{2(\gamma+1)}\right) \\
& +C_{11}\left[\left(g_{1}^{\prime} \diamond \nabla u\right)(t)+\left(g_{2}^{\prime} \diamond \nabla v\right)(t)\right] \\
& +\left\{\frac{\varepsilon_{1}}{4 \epsilon}+\varepsilon_{2}\left[\left(2 \eta+\frac{2+C^{2}}{4 \eta}\right)\left(m_{0}-l\right)+\frac{C_{6}}{4 \eta}\right]\right\} \\
& \times\left[\left(g_{1} \diamond \nabla u\right)(t)+\left(g_{2} \diamond \nabla v\right)(t)\right],
\end{aligned}
$$

which yields (if needed, one can choose $\varepsilon_{2}$ sufficiently small)

$$
\begin{array}{r}
G_{1}^{\prime}(t) \leq-c_{*} E(t)+C\left[\left(g_{1} \diamond \nabla u\right)(t)+\left(g_{2} \diamond \nabla v\right)(t)\right], \\
\forall t \geq t_{0},
\end{array}
$$

where $c_{*}$ is some positive constant. It follows from (165), (A3), and (28) that

$$
\begin{aligned}
\xi(t) G_{1}^{\prime}(t) \leq & -c_{*} \xi(t) E(t) \\
& +C \xi(t)\left[\left(g_{1} \diamond \nabla u\right)(t)+\left(g_{2} \diamond \nabla v\right)(t)\right] \\
\leq & -c_{*} \xi(t) E(t)+C \xi_{1}(t)\left(g_{1} \diamond \nabla u\right)(t) \\
& +C \xi_{2}(t)\left(g_{2} \diamond \nabla v\right)(t) \\
\leq & -c_{*} \xi(t) E(t) \\
& -C\left[\left(g_{1}^{\prime} \diamond \nabla u\right)(t)+\left(g_{2}^{\prime} \diamond \nabla v\right)(t)\right] \\
\leq & -c_{*} \xi(t) E(t)-C E^{\prime}(t), \quad \forall t \geq t_{0} .
\end{aligned}
$$

That is

$$
L_{*}^{\prime}(t) \leq-c_{*} \xi(t) E(t) \leq-k \xi(t) L_{*}(t), \quad \forall t \geq t_{0},
$$


where $L_{*}(t)=\xi(t) G_{1}(t)+C E(t)$ is equivalent to $E(t)$ due to (135) and $k$ is a positive constant. A simple integration of (167) leads to

$$
L_{*}(t) \leq L_{*}\left(t_{0}\right) e^{-k \int_{t_{0}}^{t} \xi(s) \mathrm{d} s}, \quad \forall t \geq t_{0} .
$$

This completes the proof.

\section{Conflict of Interests}

The authors declare that there is no conflict of interests regarding the publication of this paper.

\section{Acknowledgments}

The authors are grateful to the anonymous referees and the editors for their useful remarks and comments on an early version of this work. This work was partly supported by the National Natural Science Foundation of China (Grant no. 11301277), the Qing Lan Project of Jiangsu Province, and the Chinese Ministry of Finance Project (Grant no. GYHY200906006).

\section{References}

[1] R. Torrejón and J. M. Yong, "On a quasilinear wave equation with memory," Nonlinear Analysis: Theory, Methods \& Applications, vol. 16, no. 1, pp. 61-78, 1991.

[2] J. E. Muñoz Rivera, "Global solution on a quasilinear wave equation with memory," Unione Matematica Italiana B, vol. 8, no. 2, pp. 289-303, 1994.

[3] S.-T. Wu and L.-Y. Tsai, "On global existence and blow-up of solutions for an integro-differential equation with strong damping," Taiwanese Journal of Mathematics, vol. 10, no. 4, pp. 979-1014, 2006.

[4] M.-R. Li and L.-Y. Tsai, "Existence and nonexistence of global solutions of some system of semilinear wave equations," Nonlinear Analysis: Theory, Methods \& Applications, vol. 54, no. 8, pp. 1397-1415, 2003.

[5] S.-T. Wu, "Exponential energy decay of solutions for an integrodifferential equation with strong damping," Journal of Mathematical Analysis and Applications, vol. 364, no. 2, pp. 609-617, 2010.

[6] T. Matsuyama and R. Ikehata, "On global solutions and energy decay for the wave equations of Kirchhoff type with nonlinear damping terms," Journal of Mathematical Analysis and Applications, vol. 204, no. 3, pp. 729-753, 1996.

[7] K. Ono, "Blowing up and global existence of solutions for some degenerate nonlinear wave equations with some dissipation," Nonlinear Analysis: Theory, Methods \& Applications, vol. 30, no. 7, pp. 4449-4457, 1997.

[8] K. Ono, "Global existence, decay, and blowup of solutions for some mildly degenerate nonlinear Kirchhoff strings," Journal of Differential Equations, vol. 137, no. 2, pp. 273-301, 1997.

[9] K. Ono, "On global existence, asymptotic stability and blowing up of solutions for some degenerate non-linear wave equations of Kirchhoff type with a strong dissipation," Mathematical Methods in the Applied Sciences, vol. 20, no. 2, pp. 151-177, 1997.

[10] K. Ono, "On global solutions and blow-up solutions of nonlinear Kirchhoff strings with nonlinear dissipation," Journal of
Mathematical Analysis and Applications, vol. 216, no. 1, pp. 321342, 1997.

[11] J. Y. Park and J. J. Bae, "On existence of solutions of nondegenerate wave equations with nonlinear damping terms," Nihonkai Mathematical Journal, vol. 9, no. 1, pp. 27-46, 1998.

[12] A. Benaissa and S. A. Messaoudi, "Blow-up of solutions for the Kirchhoff equation of $q$-Laplacian type with nonlinear dissipation," Colloquium Mathematicum, vol. 94, no. 1, pp. 103109, 2002.

[13] S.-T. Wu and L.-Y. Tsai, "On a system of nonlinear wave equations of Kirchhoff type with a strong dissipation," Tamkang Journal of Mathematics, vol. 38, no. 1, pp. 1-20, 2007.

[14] M. M. Cavalcanti, V. N. Domingos Cavalcanti, and J. A. Soriano, "Exponential decay for the solution of semilinear viscoelastic wave equations with localized damping," Electronic Journal of Differential Equations, vol. 2002, no. 44, 14 pages, 2002.

[15] E. Zuazua, "Exponential decay for the semilinear wave equation with locally distributed damping," Communications in Partial Differential Equations, vol. 15, no. 2, pp. 205-235, 1990.

[16] M. M. Cavalcanti and H. P. Oquendo, "Frictional versus viscoelastic damping in a semilinear wave equation," SIAM Journal on Control and Optimization, vol. 42, no. 4, pp. 1310-1324, 2003.

[17] S. A. Messaoudi, "Blow up and global existence in a nonlinear viscoelastic wave equation," Mathematische Nachrichten, vol. 260, pp. 58-66, 2003.

[18] S. A. Messaoudi, "Blow-up of positive-initial-energy solutions of a nonlinear viscoelastic hyperbolic equation," Journal of Mathematical Analysis and Applications, vol. 320, no. 2, pp. 902915, 2006.

[19] W. Liu, "Global existence, asymptotic behavior and blow-up of solutions for a viscoelastic equation with strong damping and nonlinear source," Topological Methods in Nonlinear Analysis, vol. 36, no. 1, pp. 153-178, 2010.

[20] X. Han and M. Wang, "Global existence and blow-up of solutions for a system of nonlinear viscoelastic wave equations with damping and source," Nonlinear Analysis: Theory, Methods \& Applications, vol. 71, no. 11, pp. 5427-5450, 2009.

[21] S. A. Messaoudi and B. Said-Houari, "Global nonexistence of positive initial-energy solutions of a system of nonlinear viscoelastic wave equations with damping and source terms," Journal of Mathematical Analysis and Applications, vol. 365, no. 1, pp. 277-287, 2010.

[22] F. Liang and H. Gao, "Exponential energy decay and blowup of solutions for a system of nonlinear viscoelastic wave equations with strong damping," Boundary Value Problems, vol. 2011, article 22, 19 pages, 2011.

[23] G. Li, L. Hong, and W. Liu, "Exponential energy decay of solutions for a system of viscoelastic wave equations of Kirchhoff type with strong damping," Applicable Analysis, vol. 92, no. 5, pp. 1046-1062, 2013.

[24] D. Andrade and L. H. Fatori, "The nonlinear transmission problem with memory," Boletim da Sociedade Paranaense de Matemática, vol. 22, no. 1, pp. 106-118, 2004.

[25] M. M. Cavalcanti, V. N. Domingos Cavalcanti, J. S. Prates Filho, and J. A. Soriano, "Existence and exponential decay for a Kirchhoff-Carrier model with viscosity," Journal of Mathematical Analysis and Applications, vol. 226, no. 1, pp. 40-60, 1998.

[26] M. M. Cavalcanti, V. N. Domingos Cavalcanti, and M. L. Santos, "Existence and uniform decay rates of solutions to a degenerate system with memory conditions at the boundary," Applied Mathematics and Computation, vol. 150, no. 2, pp. 439465, 2004. 
[27] V. Komornik, Exact Controllability and Stabilization, Research in Applied Mathematics, Masson, Paris, France, 1994.

[28] W. Liu, "General decay rate estimate for a viscoelastic equation with weakly nonlinear time-dependent dissipation and source terms," Journal of Mathematical Physics, vol. 50, no. 11, Article ID 113506, 17 pages, 2009.

[29] J. Y. Park and J. J. Bae, "Variational inequality for quasilinear wave equations with nonlinear damping terms," Nonlinear Analysis: Theory, Methods \& Applications, vol. 50, no. 8, pp. 1065-1083, 2002.

[30] W. Liu, "General decay and blow-up of solution for a quasilinear viscoelastic problem with nonlinear source," Nonlinear Analysis: Theory, Methods \& Applications, vol. 73, no. 6, pp. 1890-1904, 2010.

[31] W. Liu and J. Yu, "On decay and blow-up of the solution for a viscoelastic wave equation with boundary damping and source terms," Nonlinear Analysis: Theory, Methods \& Applications, vol. 74, no. 6, pp. 2175-2190, 2011.

[32] B. Said-Houari, "Global nonexistence of positive initial-energy solutions of a system of nonlinear wave equations with damping and source terms," Differential and Integral Equations, vol. 23, no. 1-2, pp. 79-92, 2010.

[33] E. Vitillaro, "Global nonexistence theorems for a class of evolution equations with dissipation," Archive for Rational Mechanics and Analysis, vol. 149, no. 2, pp. 155-182, 1999.

[34] S.-T. Wu, "Blow-up of solutions for a system of nonlinear wave equations with nonlinear damping," Electronic Journal of Differential Equations, vol. 2009, no. 105, 11 pages, 2009.

[35] K. Agre and M. A. Rammaha, "Systems of nonlinear wave equations with damping and source terms," Differential and Integral Equations, vol. 19, no. 11, pp. 1235-1270, 2006.

[36] M.-R. Li and L.-Y. Tsai, "On a system of nonlinear wave equations," Taiwanese Journal of Mathematics, vol. 7, no. 4, pp. 557-573, 2003.

[37] W. Liu, "Uniform decay of solutions for a quasilinear system of viscoelastic equations," Nonlinear Analysis: Theory, Methods \& Applications, vol. 71, no. 5-6, pp. 2257-2267, 2009.

[38] F. Sun and M. Wang, "Global and blow-up solutions for a system of nonlinear hyperbolic equations with dissipative terms," Nonlinear Analysis: Theory, Methods \& Applications, vol. 64, no. 4, pp. 739-761, 2006.

[39] S.-T. Wu, "On decay and blow-up of solutions for a system of nonlinear wave equations," Journal of Mathematical Analysis and Applications, vol. 394, no. 1, pp. 360-377, 2012.

[40] S. Yu, "Polynomial stability of solutions for a system of nonlinear viscoelastic equations," Applicable Analysis, vol. 88, no. 7, pp. 1039-1051, 2009.

[41] R. A. Adams, Sobolev Spaces, vol. 65 of Pure and Applied Mathematics, Academic Press, New York, NY, USA, 1975.

[42] S. A. Messaoudi, "General decay of the solution energy in a viscoelastic equation with a nonlinear source," Nonlinear Analysis: Theory, Methods \& Applications, vol. 69, no. 8, pp. 2589-2598, 2008.

[43] H. A. Levine, "Some nonexistence and instability theorems for solutions of formally parabolic equations of the form $P u_{t t}=$ $-A u+\mathscr{F}(u)$," Archive for Rational Mechanics and Analysis, vol. 51, pp. 371-386, 1973.

[44] H. A. Levine, "Instability and nonexistence of global solutions to nonlinear wave equations of the form $P u_{t t}=-A u+\mathscr{F}(u)$," Transactions of the American Mathematical Society, vol. 192, pp. 1-21, 1974. 


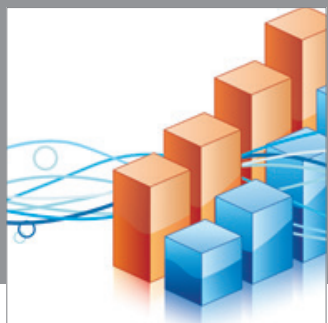

Advances in

Operations Research

mansans

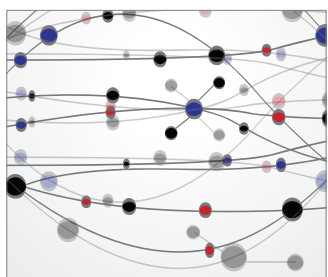

The Scientific World Journal
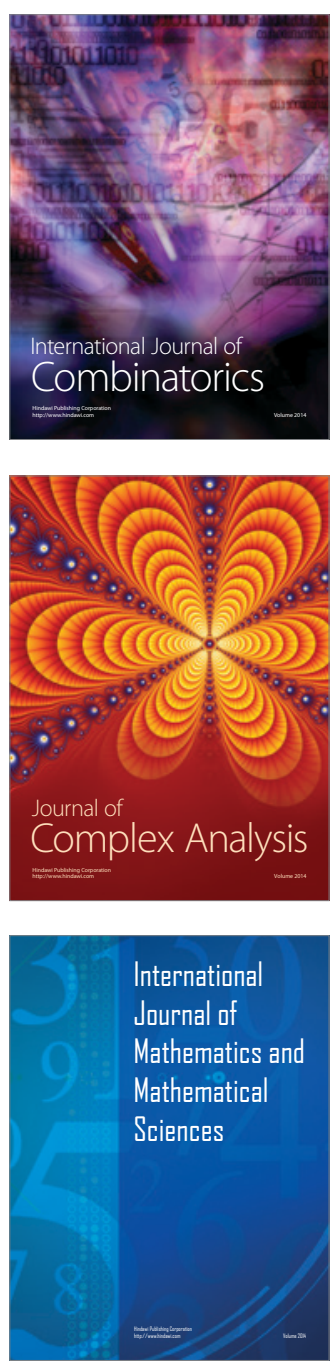
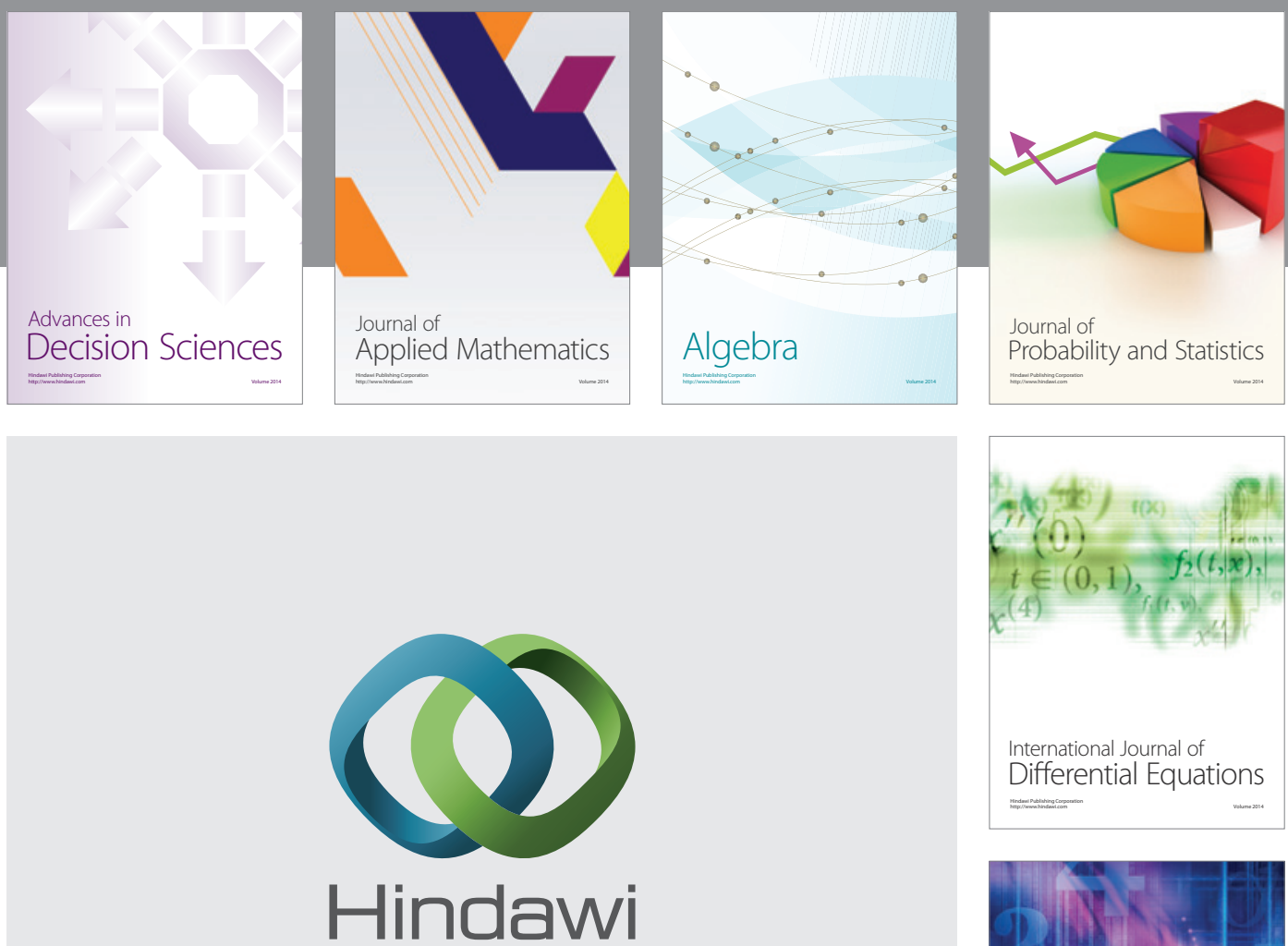

Submit your manuscripts at http://www.hindawi.com
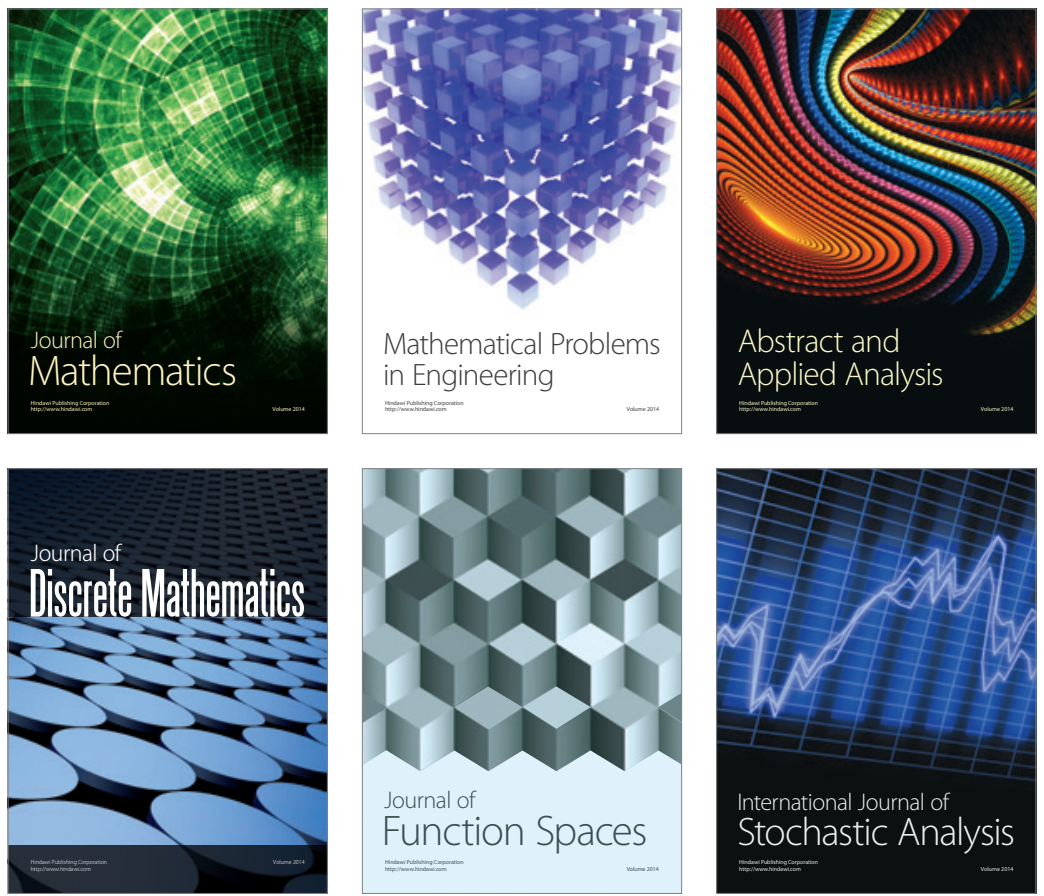

Journal of

Function Spaces

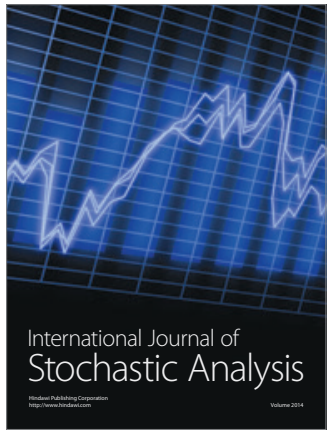

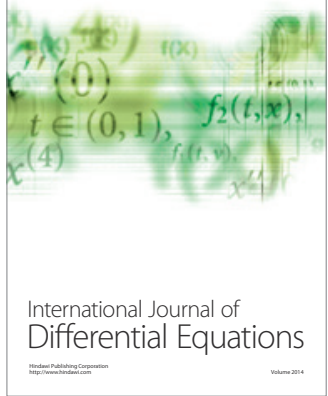
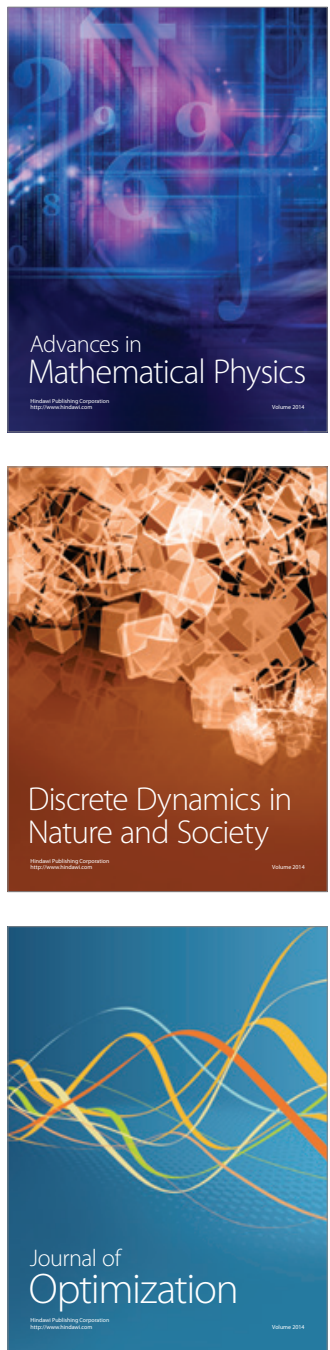